\title{
Energy Harvesting for Internet of Things with Heterogeneous Users
}

\author{
Desheng Wang, ${ }_{1}^{1}$ Haizhen Liu, ${ }^{1}$ Xiaoqiang Ma, ${ }^{1}$ Jun Wang, \\ Yanrong Peng, ${ }^{1}$ and Yanyan $\mathrm{Wu}^{2}$ \\ ${ }^{1}$ School of EIC, Huazhong University of Science and Technology, Wuhan, China \\ ${ }^{2}$ School of Public Management, South-Central University for Nationalities, Wuhan, China \\ Correspondence should be addressed to Xiaoqiang Ma; maxiaoqiang@hust.edu.cn
}

Received 17 March 2017; Accepted 11 June 2017; Published 30 July 2017

Academic Editor: Pierre-Martin Tardif

Copyright (C) 2017 Desheng Wang et al. This is an open access article distributed under the Creative Commons Attribution License, which permits unrestricted use, distribution, and reproduction in any medium, provided the original work is properly cited.

We study the energy harvesting problem in the Internet of Things with heterogeneous users, where there are three types of single-antenna users: ID users that only receive information, EH users that can only receive energy, and ID/EH users that receive information and energy simultaneously from a multiantenna base station via power splitting. We aim to maximize the minimum signal-to-interference-plus-noise ratio (SINR) of the ID users and ID/EH users by jointly designing the power allocation at the transmitter and the power splitting strategy at the ID/EH receivers under the maximum transmit power and the minimum energy harvesting constraints. Specifically, we first apply the semidefinite relaxation (SDR), zero-forcing (ZF), and maximum ratio transmission (MRT) techniques to solve the nonconvex problems. We then apply the zero-forcing dirty paper coding (ZF-DPC) technique to eliminate the multiuser interference and derive the closed-form optimal solution. Numerical results show that ZF-DPC provides higher achievable minimum SINR than SDR and ZF in most cases.

\section{Introduction}

Recently, the Internet of Things (IoT) [1-3] has been invading various industries. Given the fact that IoT normally consists of resource-constrained devices and relies on wireless communication for data transmission, energy efficiency is an important issue. For example, in wireless sensor networks (WSNs), a key component and enabler of IoT, the sensor nodes are normally powered by batteries that have very limited lifetime. The network lifetime can be extended by replacing or recharging the batteries, which, however, are inconvenient, costly, and environmentally unfriendly $[4,5]$. As an alternative solution to prolonging the network lifetime, energy harvesting [6] has become an appealing solution that potentially provides unlimited power supply to wireless networks by scavenging energy from the environment. The radio frequency (RF) signals emitted by ambient transmitters are a viable source for wireless energy harvesting (EH). As an emerging energy harvesting technique, simultaneous wireless information and power transfer (SWIPT) [7] has drawn an upsurge of interests, where RF signals are used to charge users' devices wirelessly. Through power splitting (PS) $[8,9]$, users are provided with information decoding (ID) and energy harvesting simultaneously, which brings great convenience.

How to get the trade-off between the achievable information and energy harvesting is still a challenging problem, and various techniques have been developed, for example, zero-forcing (ZF), maximum ratio transmission (MRT), and semidefinite relaxation (SDR) [10-14]. It is worth noting that most existing studies only consider $I D / E H$ users that can receive information and energy simultaneously. Yet, in practice, there are still many ID users that can only transmit/receive information and do not have the ability to harvest energy as well as EH users that only harvest energy from RF signals, which should be also considered in a unified framework. Hence, in this paper, we, for the first time, consider the coexistence of all the three types of users under multiuser MISO broadcast channels. Our aim is to maximize 
the minimum SINR of all users except for the EH users by jointly designing the power allocations at the transmitter and the PS ratios for ID/EH users who need to harvest energy under both the maximum transmit power and the minimum $\mathrm{EH}$ constraints.

In the remainder of this paper, we first present the system model and problem formulation and prove the feasibility of the problem. We then apply three traditional techniques, namely, semidefinite relaxation (SDR), zero-forcing (ZF), and maximum ratio transmission (MRT), to solve the problem. Moreover, it is well known that dirty paper coding (DPC), a nonlinear precoding scheme, can precancel noncausal interference without loss of information and also achieve the capacity region for MIMO broadcast channels (BCs) [15], which, however, has a relatively high computational complexity. To this end, we develop a suboptimal yet efficient solution based on zero-forcing dirty paper coding (ZFDPC) [16]. We compare the three schemes through extensive simulations, and the results show that ZF-DPC provides better performance than SDR and ZF in most cases.

\section{Related Work}

Initial research works in the field of IoT mainly focus on the building management $[17,18]$ and the related security as well as energy issues [19]. Building management includes many aspects, for example, traffic control, surveillance, energy management, and indoor environmental and air quality (IEAQ) control. The authors in [3] propose an energyefficient large-scale and diffusive object monitoring mechanism to reduce communication costs and thus to reduce the energy consumption. In this paper, we propose using SWIPT for energy harvesting in IoT, which is convenient, costless, and environmentally friendly.

SWIPT has drawn an upsurge of interests. The authors in [20] study a multiuser multiple-input single-output (MISO) broadcast SWIPT system, where there exist two kinds of users, namely, ID users and EH users; the aim is to maximize the weighted sum power transferred to all $\mathrm{EH}$ receivers subject to given minimum SINR constraints at different ID users. The authors in [21] consider a point-to-point wireless link over the narrow band flat-fading channel subject to timevarying cochannel interference and then propose two different schemes, namely, time switching (TS) and power splitting (PS), for distributed information and energy receivers to evaluate the performance of the system. The recent research mainly focuses on the users who can receive information and harvest energy simultaneously. The authors in [22] consider SWIPT in the multiuser single-input single-output (SISO) interference channels and maximize the minimum SINR of users under the maximum transmit power and the minimum $\mathrm{EH}$ constraints; then the authors propose two algorithms: a centralized algorithm to optimally solve the nonconvex problem and a distributed algorithm designed to update its transmit power and PS ratio through an iterative process. The authors in [10] utilize the ZF and MRT schemes to minimize the total transmit power at the BS under the given SINR and

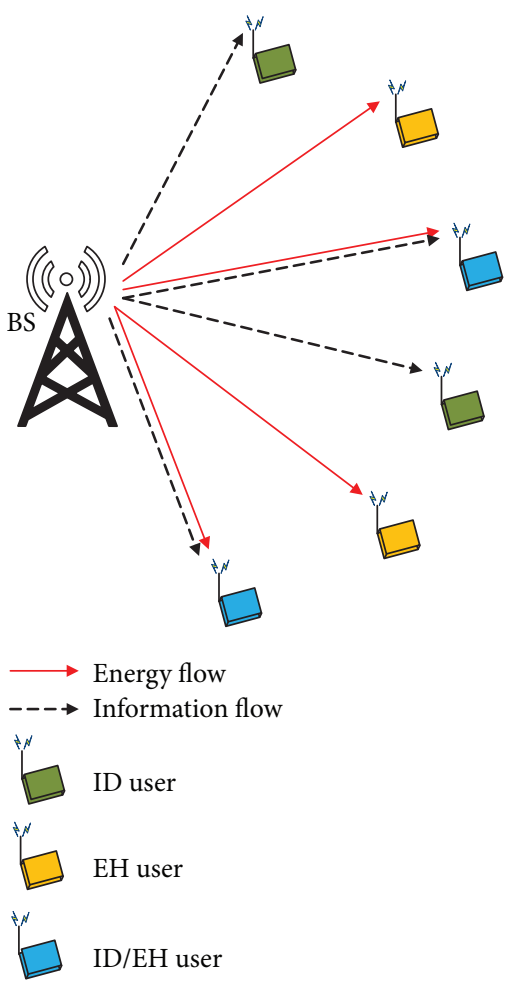

Figure 1: A general model for the coexistence of heterogeneous users.

the minimum EH constraints. The authors in [11-13] apply the SDR technique [14] to solve the nonconvex problem.

Different from most of the existing studies that only consider ID/EH users, the novelty of our work is to investigate the coexistence of ID users, ID/EH users, and EH users under the multiuser MISO broadcast channels, as shown in Figure 1. Our objective is to maximize the minimum SINR of the ID and ID/EH users by jointly designing the power allocations at the transmitter and the PS ratios. Specifically, we discuss how to adapt the traditional schemes in the literature such as SDR, ZF, and MRT to the new scenario and examine their effectiveness. We further propose a new nonlinear ZFDPC scheme for the new scenario and compare it with the traditional ones.

\section{System Model and Problem Formulation}

We consider a multiuser MISO broadcast system consisting of one BS and $K$ users. The system model is depicted in Figure 2, where the BS is equipped with $N_{t}>1$ antennas, and each user is equipped with one antenna. We summarized the notations in Notations. The first $M$ users can only receive information (namely, ID users), while the next $N$ users can receive information and harvest energy through a power splitter simultaneously (namely, ID/EH users), and the other $(K-M-N)$ users can only harvest energy (namely, EH users). We assume linear transmit precoding at the BS, where each 


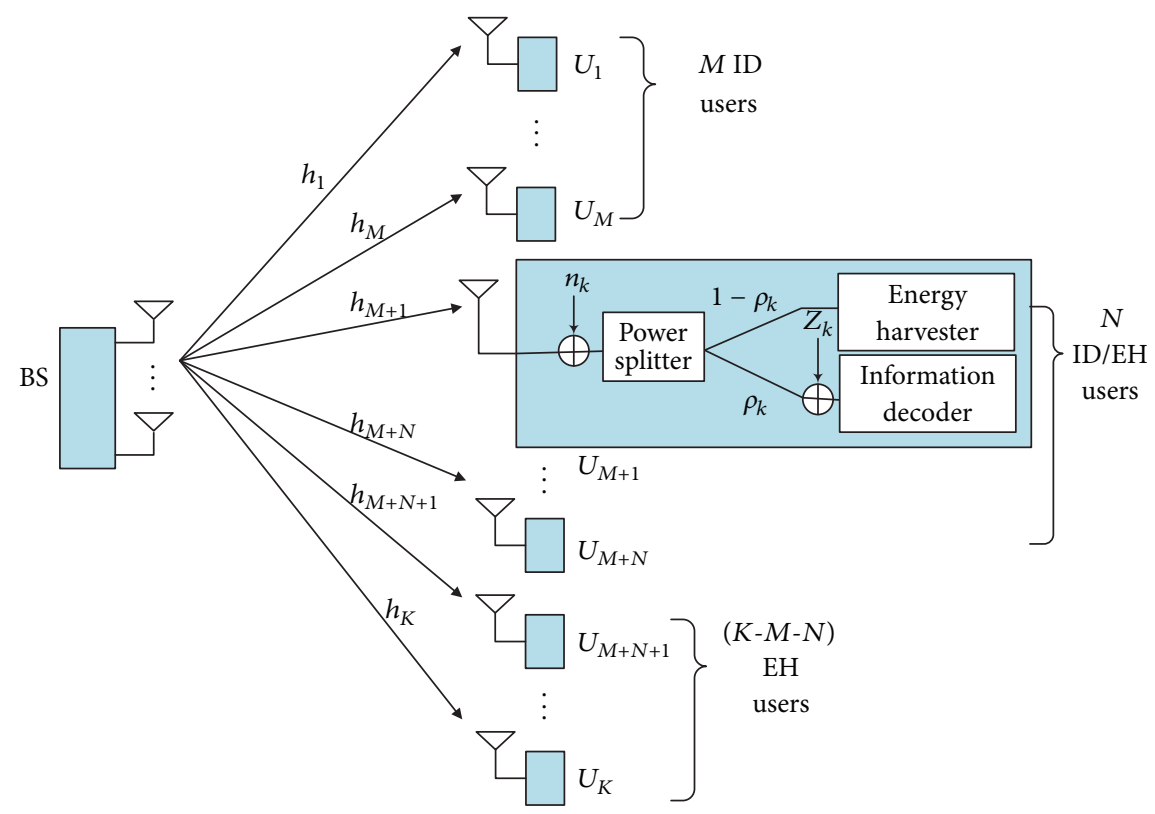

Figure 2: A multiuser MISO broadcast system.

user is assigned one dedicated information beam [14]. The complex baseband transmitted signal at the BS is

$$
\mathbf{x}=\sum_{k=1}^{K} \mathbf{v}_{k} s_{k}
$$

where $s_{k}$ denotes the transmitted data symbol for user $k$ and $\mathbf{v}_{k}$ is the corresponding transmit beamforming vector. It is assumed that $s_{k}, k=1, \ldots, K$, are independent and identically distributed (i.i.d.) cyclic symmetric complex Gaussian (CSCG) random variables with zero mean and unit variance, denoted by $s_{k} \sim \mathscr{C} \mathscr{N}(0,1)$.

We assume quasi-static flat-fading channel for all users and for convenience denote $\mathbf{h}_{k}$ as the conjugated complex channel vector from BS to user $k$. Then the received signal at user $k$ is given by

$$
y_{k}=\mathbf{h}_{k}^{H} \sum_{j=1}^{K} \mathbf{v}_{j} s_{j}+n_{k}, \quad k=1, \ldots, K,
$$

where $n_{k} \sim \mathscr{C} \mathscr{N}\left(0, \sigma_{k}^{2}\right)$ denotes the antenna noise at user $k$. Therefore, the SINR of the first $M$ ID users is

$$
\operatorname{SINR}_{k}=\frac{\left|\mathbf{h}_{k}^{H} \mathbf{v}_{k}\right|^{2}}{\sum_{j \neq k}\left|\mathbf{h}_{k}^{H} \mathbf{v}_{j}\right|^{2}+\sigma_{k}^{2}}, \quad k=1, \ldots, M .
$$

As for the next $N$ ID/EH users, we assume that each applies the PS scheme [8] to coordinate the process of information decoding and energy harvesting from the received signal, which allocates a $\rho_{k}\left(0 \leq \rho_{k} \leq 1\right)$ portion of the signal power to ID and the remaining $\left(1-\rho_{k}\right)$ portion to EH. As a result, the signal split to user $k$ 's ID part is

$$
\begin{aligned}
y_{k}^{\mathrm{ID}}=\sqrt{\rho_{k}}\left(\mathbf{h}_{k}^{H} \sum_{j=1}^{K} \mathbf{v}_{j} s_{j}+n_{k}\right)+ & z_{k}, \\
& k=M+1, \ldots, M+N,
\end{aligned}
$$

where $z_{k} \sim \mathscr{C} \mathscr{N}\left(0, \sigma_{z}^{2}\right)$ is the additional noise introduced by user $k$ 's ID part.

Accordingly, the SINR at user $k$ 's ID part is given by

$$
\begin{aligned}
\operatorname{SINR}_{k}=\frac{\rho_{k}\left|\mathbf{h}_{k}^{H} \mathbf{v}_{k}\right|^{2}}{\rho_{k} \sum_{j \neq k}\left|\mathbf{h}_{k}^{H} \mathbf{v}_{j}\right|^{2}+\rho_{k} \sigma_{k}^{2}+\sigma_{z}^{2}} & \\
& k=M+1, \ldots, M+N .
\end{aligned}
$$

On the other hand, the signal split to user $k$ 's EH part is

$$
\begin{aligned}
y_{k}^{\mathrm{EH}}=\sqrt{1-\rho_{k}}\left(\mathbf{h}_{k}^{H} \sum_{j=1}^{K} \mathbf{v}_{j} s_{j}+n_{k}\right), & \\
k & =M+1, \ldots, M+N .
\end{aligned}
$$

Then, the harvested power of user $k$ 's EH part is given by

$$
\begin{aligned}
E_{k}=\zeta_{k}\left(1-\rho_{k}\right)\left(\sum_{j=1}^{K}\left|\mathbf{h}_{k}^{H} \mathbf{v}_{j}\right|^{2}+\sigma_{k}^{2}\right), & \\
k & =M+1, \ldots, M+N,
\end{aligned}
$$

where $\zeta_{k} \in(0,1]$ denotes the energy conversion efficiency of user $k$. 
For the remaining $(K-M-N) \mathrm{EH}$ users, we have

$E_{k}=\zeta_{k}\left(\sum_{j=1}^{K}\left|\mathbf{h}_{k}^{H} \mathbf{v}_{j}\right|^{2}+\sigma_{k}^{2}\right), \quad k=M+N+1, \ldots, K$

Assume that each EH user $k(k=M+1, \ldots, K)$ has a minimum harvest energy demand $e_{k}$ and that the BS has a maximum transmit power constraint $P^{\max }$. Our aim is to maximize the minimum SINR by jointly designing the transmit beamforming vectors $\left\{\mathbf{v}_{k}\right\}, k=1, \ldots, K$, and the PS ratios $\left\{\rho_{k}\right\}, k=M+1, \ldots, M+N$, under these constraints, which is formulated as follows:

$$
\begin{array}{ll}
\max _{\mathbf{v}, \boldsymbol{\rho}} & \min _{k=1, \ldots, M+N} \operatorname{SINR}_{k} \\
\text { s.t. } & \sum_{k=1}^{K}\left\|\mathbf{v}_{k}\right\|^{2} \leq P^{\max }, \\
& E_{k} \geq e_{k}, \quad k=M+1, \ldots, K, \\
& 0 \leq \rho_{k} \leq 1, \quad k=M+1, \ldots, M+N,
\end{array}
$$

where

$$
\begin{array}{rl}
\operatorname{SINR}_{k} & =\frac{\left|\mathbf{h}_{k}^{H} \mathbf{v}_{k}\right|^{2}}{\sum_{j \neq k}\left|\mathbf{h}_{k}^{H} \mathbf{v}_{j}\right|^{2}+\sigma_{k}^{2}}, \quad k=1, \ldots, M, \\
\operatorname{SINR}_{k} & =\frac{\rho_{k}\left|\mathbf{h}_{k}^{H} \mathbf{v}_{k}\right|^{2}}{\rho_{k} \sum_{j \neq k}\left|\mathbf{h}_{k}^{H} \mathbf{v}_{j}\right|^{2}+\rho_{k} \sigma_{k}^{2}+\sigma_{z}^{2}}, \\
& k=M+1, \ldots, M+N, \\
E_{k} & =\zeta_{k}\left(1-\rho_{k}\right)\left(\sum_{j=1}^{K}\left|\mathbf{h}_{k}^{H} \mathbf{v}_{j}\right|^{2}+\sigma_{k}^{2}\right), \\
k & =M+1, \ldots, M+N, \\
E_{k} & =\zeta_{k}\left(\sum_{j=1}^{K}\left|\mathbf{h}_{k}^{H} \mathbf{v}_{j}\right|^{2}+\sigma_{k}^{2}\right), \\
k & k M+N+1, \ldots, K .
\end{array}
$$

By introducing an auxiliary variable $\tau=$ $\min _{k=1, \ldots, M+N} \mathrm{SINR}_{k}$, problem (9) can be reformulated as

$$
\begin{array}{ll}
\max _{\mathbf{v}, \boldsymbol{\rho}, \tau} & \tau \\
\text { s.t. } & \operatorname{SINR}_{k} \geq \tau, \quad k=1, \ldots, M+N, \\
& \sum_{k=1}^{K}\left\|\mathbf{v}_{k}\right\|^{2} \leq P^{\max }, \\
& E_{k} \geq e_{k}, \quad k=M+1, \ldots, K, \\
& 0 \leq \rho_{k} \leq 1, \quad k=M+1, \ldots, M+N .
\end{array}
$$

It can be shown that problem (11) becomes a feasibility problem for any given $\tau$ (please refer to Appendix A for details). Therefore, by applying the bisection search method on $\tau$ over a specific interval and solving the feasibility problem at each step with the associated $\tau$, the optimal solution to problem (11) is also that to problem (9).

\section{Three Traditional Schemes: SDR, $Z F$, and MRT}

In this section, we introduce three traditional schemes, namely, SDR, ZF, and MRT, to solve the nonconvex problem (11) in a multiuser MISO broadcast system.

4.1. SDR Scheme. Define $\mathbf{X}_{k}=\mathbf{v}_{k} \mathbf{v}_{k}^{H}, \forall k$; then $\operatorname{rank}\left(\mathbf{X}_{k}\right)=1$, $\forall k$. By ignoring the rank-one constraint for all $\mathbf{X}_{k}$, the SDR of problem (11) is given by

$$
\begin{array}{ll}
\max _{\mathbf{X}, \boldsymbol{\rho}, \tau} & \tau \\
\text { s.t. } & \sum_{k=1}^{K} \operatorname{trace}\left(\mathbf{X}_{k}\right) \leq P^{\max }, \\
& \frac{\mathbf{h}_{k}^{H} \mathbf{X}_{k} \mathbf{h}_{k}}{\sum_{j \neq k} \mathbf{h}_{k}^{H} \mathbf{X}_{j} \mathbf{h}_{k}+\sigma_{k}^{2}} \geq \tau, \quad k=1, \ldots, M, \\
& \frac{\rho_{k} \mathbf{h}_{k}^{H} \mathbf{X}_{k} \mathbf{h}_{k}}{\rho_{k} \sum_{j \neq k} \mathbf{h}_{k}^{H} \mathbf{X}_{j} \mathbf{h}_{k}+\rho_{k} \sigma_{k}^{2}+\sigma_{z}^{2}} \geq \tau, \\
& \zeta_{k}\left(1-\rho_{k}\right)\left(\sum_{j=1}^{K} \mathbf{h}_{k}^{H} \mathbf{X}_{j} \mathbf{h}_{k}+\sigma_{k}^{2}\right) \geq e_{k}, \\
& \\
& 0 \leq \rho_{k} \leq 1, \quad k=M+1, \ldots, M+N, N, \\
& \left(\sum_{j=1}^{K} \mathbf{h}_{k}^{H} \mathbf{X}_{j} \mathbf{h}_{k}+\sigma_{k}^{2}\right) \geq e_{k}, \\
& k=1, \ldots, M+N,
\end{array}
$$

$\mathbf{X}_{k} \succeq 0, \quad \forall k$.

Problem (12) is still nonconvex, since both the SINR and harvested power constraints involve coupled $\mathbf{X}_{k}$ and $\rho_{k}$. This problem can be reformulated as the following problem:

$$
\begin{aligned}
\max _{\mathbf{X}, \boldsymbol{\rho}, \tau} & \tau \\
\text { s.t. } & \sum_{k=1}^{K} \operatorname{trace}\left(\mathbf{X}_{k}\right) \leq P^{\max }, \\
& \frac{1}{\tau} \mathbf{h}_{k}^{H} \mathbf{X}_{k} \mathbf{h}_{k}-\sum_{j \neq k} \mathbf{h}_{k}^{H} \mathbf{X}_{j} \mathbf{h}_{k} \geq \sigma_{k}^{2}, \quad k=1, \ldots, M,
\end{aligned}
$$




$$
\begin{gathered}
\frac{1}{\tau} \mathbf{h}_{k}^{H} \mathbf{X}_{k} \mathbf{h}_{k}-\sum_{j \neq k} \mathbf{h}_{k}^{H} \mathbf{X}_{j} \mathbf{h}_{k} \geq \sigma_{k}^{2}+\frac{\sigma_{z}^{2}}{\rho_{k}}, \\
k=M+1, \ldots, M+N, \\
\sum_{j=1}^{K} \mathbf{h}_{k}^{H} \mathbf{X}_{j} \mathbf{h}_{k} \geq \frac{e_{k}}{\zeta_{k}\left(1-\rho_{k}\right)}-\sigma_{k}^{2}, \\
k=M+1, \ldots, M+N, \\
0 \leq \rho_{k} \leq 1, \quad k=M+1, \ldots, M+N, \\
\sum_{j=1}^{K} \mathbf{h}_{k}^{H} \mathbf{X}_{j} \mathbf{h}_{k} \geq \frac{e_{k}}{\zeta_{k}}-\sigma_{k}^{2}, \\
k=M+1, \ldots, M+N,
\end{gathered}
$$

$\mathbf{X}_{k} \geq 0, \quad \forall k$

Note that problem (13) is convex; therefore, the optimal solution can be obtained by applying the bisection search method on $\tau$. Let $\left\{\mathbf{X}_{k}^{*}\right\}$ and $\left\{\rho_{k}^{*}\right\}$ denote the optimal solution to problem (13). If $\left\{\mathbf{X}_{k}^{*}\right\}$ satisfies $\operatorname{rank}\left(\mathbf{X}_{k}^{*}\right)=1, \forall k$, then the optimal beamforming solution to problem (11) can be obtained by doing eigenvalue decomposition (EVD) of $\mathbf{X}_{k}^{*}=$ $\overline{\mathbf{v}}_{k} \overline{\mathbf{v}}_{k}^{H}, \forall k$; otherwise we need to do Gaussian randomization [23] to choose the best solution.

In Appendix $\mathrm{B}$, we have proven that $\mathbf{X}_{k}^{*}$ satisfies $\operatorname{rank}\left(\mathbf{X}_{k}^{*}\right)=1$ when there are no EH users, where $K=$ $M+N$, and from the numerical analysis in Section 6, we could verify that no matter whether there exist EH users, $\mathbf{X}_{k}^{*}$ for the ID users and the ID/EH users always satisfy the rank-one conditions, but it is not suitable for the $\mathrm{EH}$ users, and thus we need to do Gaussian randomization to choose the best beamforming vectors $\left\{\mathbf{v}_{k}\right\}$ for them. However, we just need to know the achievable minimum SINR which is obtained directly from $\mathbf{X}_{k}^{*}$ rather than $\left\{\mathbf{v}_{k}\right\}$ in this paper, and thus we can ignore the process of Gaussian randomization.

The optimal solution to problem (11) can be obtained via solving problem (13) by the interior-point algorithm [24] using standard solvers, for example, CVX [25], and the complexity of the interior-point algorithm is $\mathcal{O}((K+$ $\left.N_{t}^{2}\right)^{3.5} \log (1 / \varepsilon)$ ) [26], where $\varepsilon$ is the desired numerical accuracy.

4.2. ZF Scheme. In ZF, the condition $N_{t} \geq K$ must be satisfied [10], and $\mathbf{h}_{k}$ are not linearly dependent. The ZF beamforming scheme can then be used to eliminate the multiuser interference by restricting $\mathbf{v}_{k}$ to satisfy $\mathbf{h}_{i}^{H} \mathbf{v}_{k}=$ $0, \forall i \neq k$. More specifically, the ZF weight $\mathbf{v}_{k}$ is provided by the solution to the following problem:

$$
\begin{array}{ll}
\min _{\mathbf{v}_{k}} & \left|\mathbf{h}_{k}^{H} \mathbf{v}_{k}\right|^{2} \\
\text { s.t. } & \mathbf{H}_{k}^{H} \mathbf{v}_{k}=\mathbf{0}_{(K-1) \times 1},
\end{array}
$$

where $\mathbf{H}_{k} \triangleq\left[\mathbf{h}_{1}, \ldots, \mathbf{h}_{k-1}, \mathbf{h}_{k+1}, \ldots, \mathbf{h}_{K}\right] \in \mathbb{C}^{N_{t} \times(K-1)}$. Assume that $\mathbf{v}_{k}=\sqrt{p_{k}} \mathbf{w}_{k}$, where $\left\|\mathbf{w}_{k}\right\|_{2}^{2}=1$ and the solution is given by [27]

$$
\mathbf{w}_{k}^{(\mathrm{ZF})}=\frac{\left(\mathbf{I}_{N_{t}}-\mathbf{F}\right) \mathbf{h}_{k}}{\left\|\left(\mathbf{I}_{N_{t}}-\mathbf{F}\right) \mathbf{h}_{k}\right\|},
$$

where $\mathbf{F}=\mathbf{H}_{k}^{\dagger} \mathbf{H}_{k}^{H}$ and $\mathbf{H}_{k}^{\dagger}=\mathbf{H}_{k}\left(\mathbf{H}_{k}^{H} \mathbf{H}_{k}\right)^{-1}$ is the MoorePenrose inverse of $\mathbf{H}_{k}$.

Let $G_{i, j}=\left|\mathbf{h}_{i}^{H} \mathbf{w}_{j}^{(\mathrm{ZF})}\right|^{2}$ denote the link gain, and we have $G_{i, j}=0, i \neq j$. Hence, problem (11) is equivalent to the following problem:

$$
\begin{array}{ll}
\max _{p_{k}, \rho, \tau} & \tau \\
\text { s.t. } & \sum_{k=1}^{K} p_{k} \leq P^{\max }, \\
& G_{k, k} p_{k} \geq \tau \sigma_{k}^{2}, \quad k=1, \ldots, M, \\
& G_{k, k} p_{k} \geq \tau \sigma_{k}^{2}+\frac{\tau \sigma_{z}^{2}}{\rho_{k}}, \quad k=M+1, \ldots, M+N, \\
& \zeta_{k}\left(1-\rho_{k}\right)\left(G_{k, k} p_{k}+\sigma_{k}^{2}\right) \geq e_{k}, \\
& \quad k=M+1, \ldots, M+N, \\
& \zeta_{k}\left(G_{k, k} p_{k}+\sigma_{k}^{2}\right) \geq e_{k}, \quad k, M+N, \\
& k=M+N+1, \ldots, K,
\end{array}
$$

$p_{k} \geq 0, \quad \forall k$.

Let $x_{k}=G_{k, k} p_{k}+\sigma_{k}^{2}, \alpha=(1+\tau) \sigma_{k}^{2}, \beta=\tau \sigma_{z}^{2}$, and $\gamma=e_{k} / \zeta_{k}$, and the closed-form solution is given by

$$
\begin{aligned}
& p_{k}^{*}=\frac{\tau \sigma_{k}^{2}}{G_{k, k}}, \quad k=1, \ldots, M, \\
& p_{k}^{*}=\frac{1}{G_{k, k}}\left(x^{*}-\sigma_{k}^{2}\right), \\
& \quad \rho_{k}^{*}=1-\frac{\gamma}{x^{*}}, k=M+1, \ldots, M+N, \\
& p_{k}^{*}=\frac{\gamma-\sigma_{k}^{2}}{G_{k, k}}, \quad k=M+N+1, \ldots, K,
\end{aligned}
$$

where $x^{*}=(1 / 2)\left(\alpha+\beta+\gamma+\sqrt{(\alpha+\beta+\gamma)^{2}-4 \alpha \gamma}\right)$. Please refer to Appendix $\mathrm{C}$ for details.

Clearly, the complexity of the ZF scheme is dominated by the $K$ times of SVD operations. Since the complexity of each SVD operation is $\mathcal{O}\left(K N_{t}^{2}+K^{2} N_{t}+K^{3}\right)$ [28], the overall complexity of the ZF scheme is $\mathcal{O}\left(K^{2} N_{t}^{2}+K^{3} N_{t}+K^{4}\right)$. 
4.3. MRT Scheme. The MRT beamforming maximizes the SNR at each receiver $\left(\left|\mathbf{h}_{k}^{H} \mathbf{v}_{k}\right|^{2} / \sigma_{k}^{2}, \forall k\right)$, and it only requires the knowledge of the direct links $\mathbf{h}_{k}$; thus, it is of relatively low complexity to obtain the beamforming vectors. Assuming that $\mathbf{v}_{k}=\sqrt{p_{k}} \mathbf{w}_{k}$, where $\left\|\mathbf{w}_{k}\right\|_{2}^{2}=1$ and $p_{k}$ is the power allocated to user $k$, the MRT beamforming [29] can be expressed as

$$
\mathbf{w}_{k}^{\mathrm{MRT}}=\frac{\mathbf{h}_{k}}{\left\|\mathbf{h}_{k}\right\|} .
$$

It is worth noting that MRT does not take into account the signals transmitted to other users and therefore it results in a strong cross-interference. Although this cross-interference is a bottleneck for conventional MISO systems, it could be beneficial for scenarios with EH constraints. Then, let $G_{i, j}=$ $\left|\mathbf{h}_{i}^{H} \mathbf{w}_{j}^{(\mathrm{MRT})}\right|^{2}$ denote the link gain; problem (11) can be cast into a second-order cone programming (SOCP) [30] formulation as follows:

$$
\begin{array}{ll}
\max _{p_{k}, \rho, \tau} & \tau \\
\text { s.t. } & \sum_{k=1}^{K} p_{k} \leq P^{\max }, \\
& \frac{1}{\tau} G_{k, k} p_{k}-\sum_{j \neq k} G_{k, j} p_{j} \geq \sigma_{k}^{2}, \quad k=1, \ldots, M, \\
& \frac{1}{\tau} G_{k, k} p_{k}-\sum_{j \neq k} G_{k, j} p_{j} \geq \sigma_{k}^{2}+\frac{\sigma_{z}^{2}}{\rho_{k}}, \\
& \quad k=M+1, \ldots, M+N, \\
& \zeta_{k}\left(1-\rho_{k}\right)\left(\sum_{j=1}^{K} G_{k, j} p_{j}+\sigma_{k}^{2}\right) \geq e_{k}, \\
& \\
& 0 \leq \rho_{k} \leq 1, \quad k=M+1, \ldots, M+N, \\
& \zeta_{k}\left(\sum_{j=1}^{K} G_{k, j} p_{j}+\sigma_{k}^{2}\right) \geq e_{k}, \\
k=M+N+1, \ldots, K, & M+N,
\end{array}
$$

$p_{k} \geq 0, \quad \forall k$

The optimal solution to problem (19) can be obtained by using standard solvers, for example, CVX. The complexity of the MRT scheme is dominated by the $K$ times of computing the beamforming vector $\mathbf{w}_{k}^{\mathrm{MRT}}$ with $\mathcal{O}(1)$ complexity each time. The SOCP algorithm for solving problem (19) is $\mathcal{O}\left((K+N)^{3.5} \log (1 / \varepsilon)\right)[26]$, where $\varepsilon$ is the preset search precision. Thus, the complexity of MRT scheme is $\mathcal{O}((K+$ $\left.N)^{3.5} \log (1 / \varepsilon)\right)$.

\section{Nonlinear Precoding Scheme: ZF-DPC}

In this section, we present the ZF-DPC scheme that applies $\mathrm{ZF}$ with $\mathrm{QR}$ decomposition to eliminate the causal interference and then uses DPC to eliminate the noncausal interference.

Denote the MISO channels by $\mathbf{H} \triangleq\left[\mathbf{h}_{1}, \mathbf{h}_{2}, \ldots, \mathbf{h}_{K}\right]^{H} \epsilon$ $\mathbb{C}^{K \times N_{t}}$. Then the QR decomposition [31] obtained by applying Gram-Schmidt orthogonalization to the rows of $\mathbf{H}^{H}$ is $\mathbf{H}^{H}=$ $\mathbf{R} \mathbf{Q}^{H}$; then $\mathbf{H}$ can be expressed as

$$
\mathbf{H}=\mathbf{Q R}^{H} \text {. }
$$

Let $m=\operatorname{rank}(\mathbf{H}) \leq \min \left\{K, N_{t}\right\}$. Then $\mathbf{Q}$ is a $K \times m$ lower triangular matrix (i.e., it has zeros above its main diagonal), and denote $q_{i, j}$ as the $(i, j)$ th element of $\mathbf{Q}$. $\mathbf{R}$ is an $N_{t} \times m$ subunitary matrix with $m$ orthonormal columns. By letting the beamforming matrix $\mathbf{B}_{\mathrm{ZF}}=\mathbf{R}$, the transmit signal of $\mathrm{ZF}$ $\mathrm{DPC}$ is given by

$$
\mathbf{x}_{\mathrm{ZF}}=\mathbf{B}_{\mathrm{ZF}} \mathbf{u}_{\mathrm{ZF}}
$$

where $\mathbf{u}_{\mathrm{ZF}}=\left[u_{1}, u_{2}, \ldots, u_{m}\right]^{T}$ and it satisfies the power constraints $\left\|\mathbf{u}_{\mathrm{ZF}}\right\|^{2} \leq P^{\max }$. Then the received signal of user $k$ is given by the set of interference channels:

$$
y_{k}=q_{k, k} u_{k}+\sum_{j<k} q_{k, j} u_{j}+n_{k}, \quad k=1, \ldots, m,
$$

while no information is sent to users $m+1, \ldots, K$. In this case, there is no point in maximizing the minimum SINR, and we assume that $\mathbf{h}_{\mathbf{k}}$ are not linearly dependent and the condition $N_{t} \geq K$ must be satisfied, and we have $m=K$.

Based on (20)-(22), the received signals $\mathbf{y}=$ $\left[y_{1}, y_{2}, \ldots, y_{K}\right]^{T}$ can be written as

$$
\mathbf{y}=\mathbf{H B}_{\mathrm{ZF}} \mathbf{u}_{\mathrm{ZF}}+\mathbf{n}=\mathbf{Q} \mathbf{u}_{\mathrm{ZF}}+\mathbf{n},
$$

where $\mathbf{n}=\left[n_{1}, n_{2}, \ldots, n_{K}\right]^{T}$. According to the QR decomposition of the channel matrix, different ordering among users will lead to different channel gain. For the sake of convenience, we assume that the coding order is $[1,2, \ldots, K]$. Note that the interuser interference from the off-diagonal entries of $\mathbf{Q}$ can be cancelled by successive DPC by the precoding matrix $\mathbf{W}=\mathbf{Q}^{-1} \operatorname{diag}\left(q_{1,1}, q_{2,2}, \ldots, q_{K, K}\right)$ and $\mathbf{u}_{\mathrm{ZF}}=$ $\mathbf{W} \overline{\mathbf{s}}_{\mathrm{ZF}}$, where $\overline{\mathbf{s}}_{\mathrm{ZF}}$ is the transmitted signal. Then, the received signals can be written as $\mathbf{y}=\operatorname{diag}\left(q_{1,1}, q_{2,2}, \ldots, q_{K, K}\right) \overline{\mathbf{s}}_{\mathrm{ZF}}+\mathbf{n}$. Thus the $\mathrm{BC}$ is transformed into $K$ independent channels with $q_{k, k}^{2}, k=1, \ldots, K$, being the channel gain. Denote $p_{k}$ as the power allocated to user $k$, and the SINR of user $k$ is given by

$$
\begin{aligned}
& \operatorname{SINR}_{k}=\frac{q_{k, k}^{2} p_{k}}{\sigma_{k}^{2}}, \quad k=1, \ldots, M, \\
& \operatorname{SINR}_{k}=\frac{\rho_{k} q_{k, k}^{2} p_{k}}{\rho_{k} \sigma_{k}^{2}+\sigma_{z}^{2}}, \quad k=M+1, \ldots, M+N .
\end{aligned}
$$


The EH of user $k$ is

$$
\begin{aligned}
& E_{k}=\zeta_{k}\left(1-\rho_{k}\right)\left(q_{k, k}^{2} p_{k}+\sigma_{k}^{2}\right), \\
& k=M+1, \ldots, M+N, \\
& E_{k}=\zeta_{k}\left(q_{k, k}^{2} p_{k}+\sigma_{k}^{2}\right), \quad k=M+N+1, \ldots, K .
\end{aligned}
$$

Now problem (11) can be reformulated as follows:

$$
\begin{array}{ll}
\max _{p_{k}, \rho, \tau} & \tau \\
\text { s.t. } & \sum_{k=1}^{K} p_{k} \leq P^{\mathrm{max}}, \\
& q_{k, k}^{2} p_{k} \geq \tau \sigma_{k}^{2}, \quad k=1, \ldots, M, \\
& q_{k, k}^{2} p_{k} \geq \tau \sigma_{k}^{2}+\frac{\tau \sigma_{z}^{2}}{\rho_{k}}, \quad k=M+1, \ldots, M+N, \\
& \zeta_{k}\left(1-\rho_{k}\right)\left(q_{k, k}^{2} p_{k}+\sigma_{k}^{2}\right) \geq e_{k}, \\
& 0 \leq \rho_{k} \leq 1, \quad k=M+1, \ldots, M+N, \\
& \zeta_{k}\left(q_{k, k}^{2} p_{k}+\sigma_{k}^{2}\right) \geq e_{k}, \quad k, M+N, \\
& p_{k} \geq 0, \quad \forall k . \quad k=M+1, \ldots, M+N,
\end{array}
$$

Similar to the ZF scheme, let $x_{k}=q_{k, k}^{2} p_{k}+\sigma_{k}^{2}, \alpha=(1+$ $\tau) \sigma_{k}^{2}, \beta=\tau \sigma_{z}^{2}$, and $\gamma=e_{k} / \zeta_{k}$, and then the solution is

$$
\begin{aligned}
& p_{k}^{*}=\frac{\tau \sigma_{k}^{2}}{q_{k, k}^{2}}, \quad k=1, \ldots, M, \\
& p_{k}^{*}=\frac{1}{q_{k, k}^{2}}\left(x^{*}-\sigma_{k}^{2}\right), \\
& \quad \rho_{k}^{*}=1-\frac{\gamma}{x^{*}}, k=M+1, \ldots, M+N, \\
& p_{k}^{*}=\frac{\gamma-\sigma_{k}^{2}}{q_{k, k}^{2}}, \quad k=M+N+1, \ldots, K,
\end{aligned}
$$

where $x^{*}=(1 / 2)\left(\alpha+\beta+\gamma+\sqrt{(\alpha+\beta+\gamma)^{2}-4 \alpha \gamma}\right)$.

The complexity of the ZF-DPC scheme is divided into two parts. The first part is to perform QR decomposition, the complexity of which is $\mathcal{O}\left(K^{2} N_{t}\right)$; the other part is to perform pseudoinverse and matrix multiplication (multiply a diagonal matrix) for both $K \times K$ matrices, and the complexity is $\mathcal{O}\left(K^{3}\right)$ [32] and $\mathcal{O}(K)$, respectively. Thus the overall complexity of the ZF-DPC scheme is $\mathcal{O}\left(K^{2} N_{t}+K^{3}\right)$.

\section{Numerical Analysis}

In this section, we numerically evaluate the performance of the proposed schemes. For simplicity, we assume that $\zeta_{k}=\zeta$, $e_{k}=e, k=M+1, \ldots, K$, and $\sigma_{c}^{2}=\sigma_{z}^{2}=10^{-4}, \forall k$; the BS is equipped with $N_{t}=8$ antennas, and the transmit power is no more than $P^{\max }$.

We have the following initial observations. First, we can see from Figure 3 that the system's performance in terms of

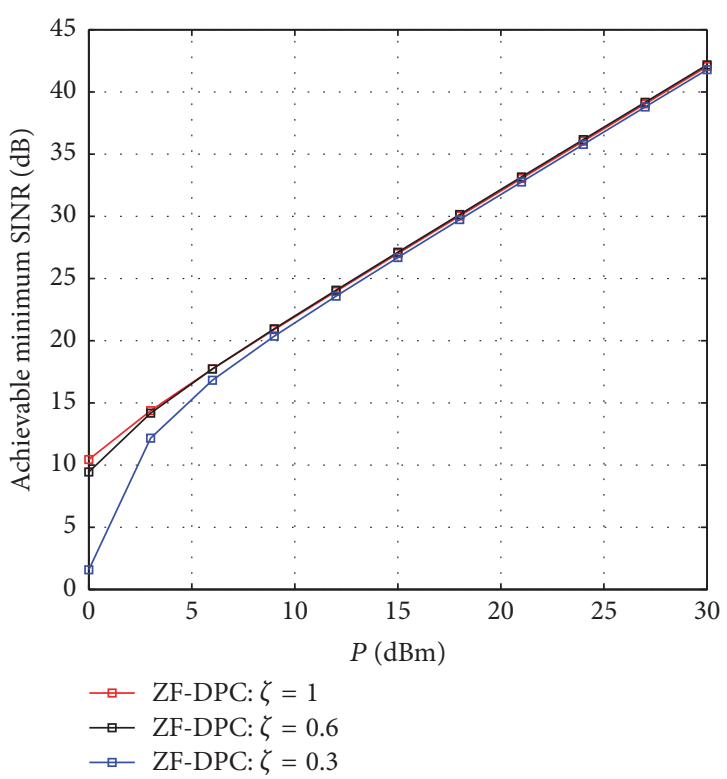

FIgURE 3: Achievable minimum SINR versus transmit power under different $\zeta$.

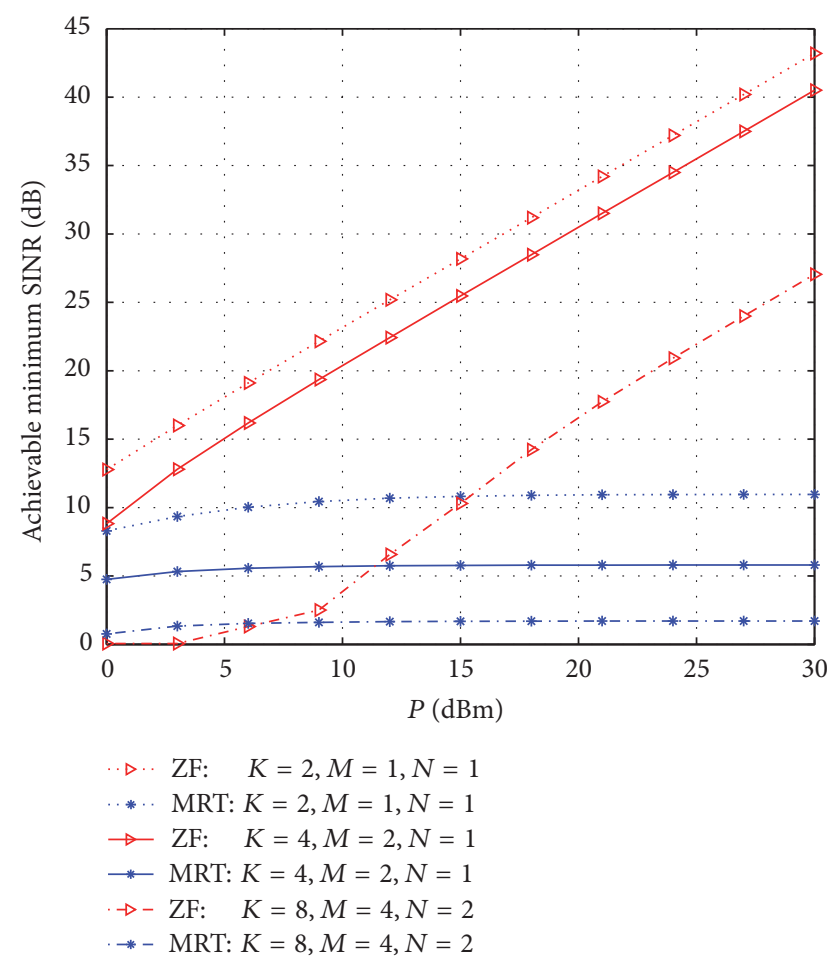

Figure 4: Comparison of ZF and MRT schemes.

the achievable minimum SINR under different $\zeta$ is almost the same when $\zeta$ exceeds a certain value, say 0.6 , in our simulation. We use $\zeta=1$ in the next simulations without loss of generality.

Second, we can see from Figure 4 that the performance of the MRT scheme remains almost unchanged when the transmit power increases, due to the strong cross-interference 


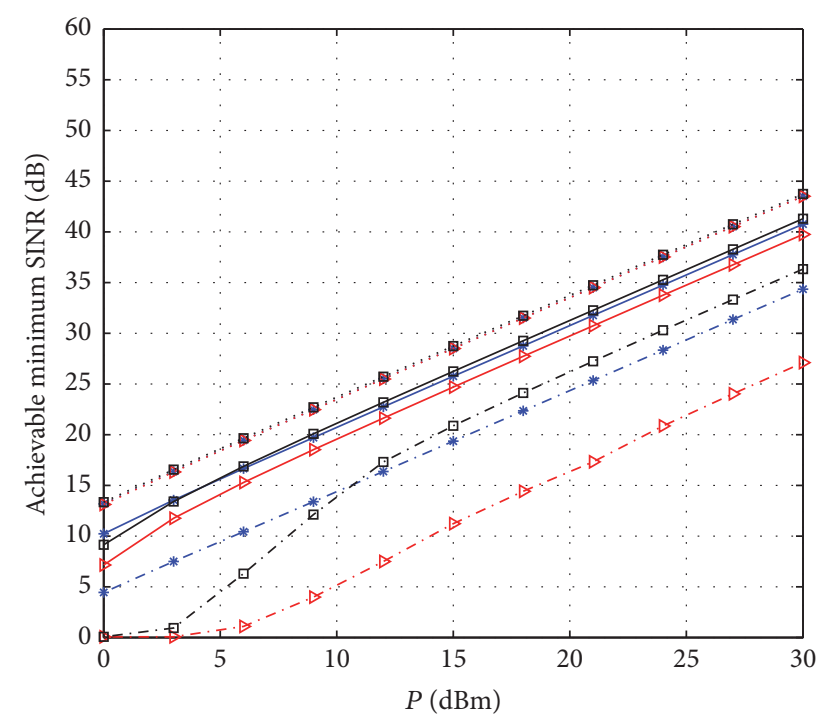

$$
\begin{aligned}
& \text { *. SDR: } K=2, M=1, N=1 \\
& \text { \. ZF: } K=2, M=1, N=1 \\
& \text {.a. ZF-DPC: } K=2, M=1, N=1 \\
& \rightarrow \text { SDR: } K=4, M=2, N=1 \\
& \rightarrow \mathrm{ZF}: \quad K=4, M=2, N=1 \\
& \rightarrow \text { ZF-DPC: } K=4, M=2, N=1 \\
& \rightarrow \text { - SDR: } \quad K=8, M=4, N=2 \\
& \rightarrow-\mathrm{ZF}: \quad K=8, M=4, N=2 \\
& \text { - } \text { - ZF-DPC: } K=8, M=4, N=2
\end{aligned}
$$

FIGURE 5: Achievable minimum SINR versus transmit power with different numbers of users.

from other users, and the ZF scheme preforms better than the MRT scheme except when $K=8, M=4$, and $N=2$ and the transmit power is relatively small, which also indicates that interference is beneficial to $\mathrm{EH}$. A simple method to ensure that the direct links are much stronger than the interference links is to strengthen the direct channel links by a multiplicative constant $\delta$ [33]; that is, $\delta \mathbf{h}_{i, i} \rightarrow \mathbf{h}_{i, i}$, which is, however, infeasible in our scenario with only one BS. We do not consider the MRT scheme in the remaining part, since it is inferior to $\mathrm{ZF}$ in most cases.

6.1. SINR versus Transmit Power. In this part, we investigate the achievable minimum SINR of the proposed schemes under different transmit power of the BS when $e=0 \mathrm{dBm}$.

From Figures 5 and 6, we can see that as the transmit power $P^{\max }$ increases from $0 \mathrm{dBm}$ to $30 \mathrm{dBm}$, the system's performance of all three schemes increases substantially, and the performance of $\mathrm{ZF}$ is always the worst. When the number of users is relatively small, the performances of the three schemes are almost the same, especially when $K=2, M=1$, and $N=1$ due to less interference from other users. Yet, as shown in Figure 5, with the increasing number of users (e.g., $K=4$ and $K=8$ ), the performances of different schemes differ significantly.

Specifically, when the transmit power is relatively small, SDR has better performance than ZF-DPC, while with higher transmit power, the performance of ZF can approach SDR, and ZF-DPC has a gain of about $2 \mathrm{~dB}$ and about $10 \mathrm{~dB}$ over SDR and ZF, respectively, when $K=8, M=4$, and $N=2$.

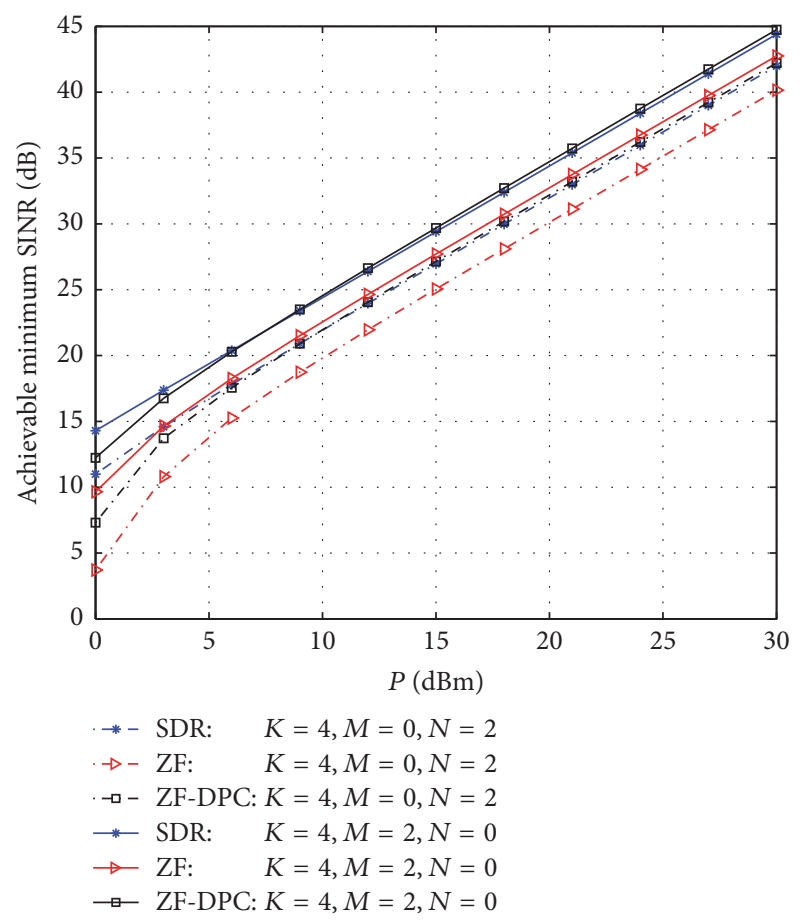

FIGURE 6: Achievable minimum SINR versus transmit power with fixed number of EH users.

We can also see from Figure 6 that when the number of total users $K$ and the number of $\mathrm{EH}$ users are fixed, with the increasing number of ID users $M$ (the number of ID/EH users decreasing correspondingly), the performances of the three schemes all improve about $3 \mathrm{~dB}$. The reason is that, with fewer energy harvesters, the BS can allocate more power to the information receivers.

Figure 7 shows that our proposed ZF-DPC technique provides higher achievable minimum SINR than SDR and ZF even for the traditional scenario with ID/EH users only.

6.2. SINR versus EH. In this part, we investigate the relationship between the achievable minimum SINR and the minimum energy harvesting of the users, with fixed transmit power of the BS $\left(P^{\max }=30 \mathrm{dBm}\right)$.

We can see from Figure 8 that the performances of the three schemes are similar when the number of users is small and the performances of different schemes differ significantly with the increasing number of the users.

From Figure 9, we can see that ZF-DPC performs worse than SDR only in the very high minimum harvest energy $e$ regions. With the increase of the ID users $M$ (the number of ID/EH users decreasing correspondingly), the performances of the three schemes all improve about $4 \mathrm{~dB}$.

Figure 10 compares the proposed schemes when there are only ID/EH users, which indicates that our proposed ZFDPC scheme is superior to the other schemes in most cases.

All the three figures again indicate the trade-off between energy harvesting and information transmission, which is a fundamental issue in the power splitting design. When the users need to harvest more energy, the achievable SINR 

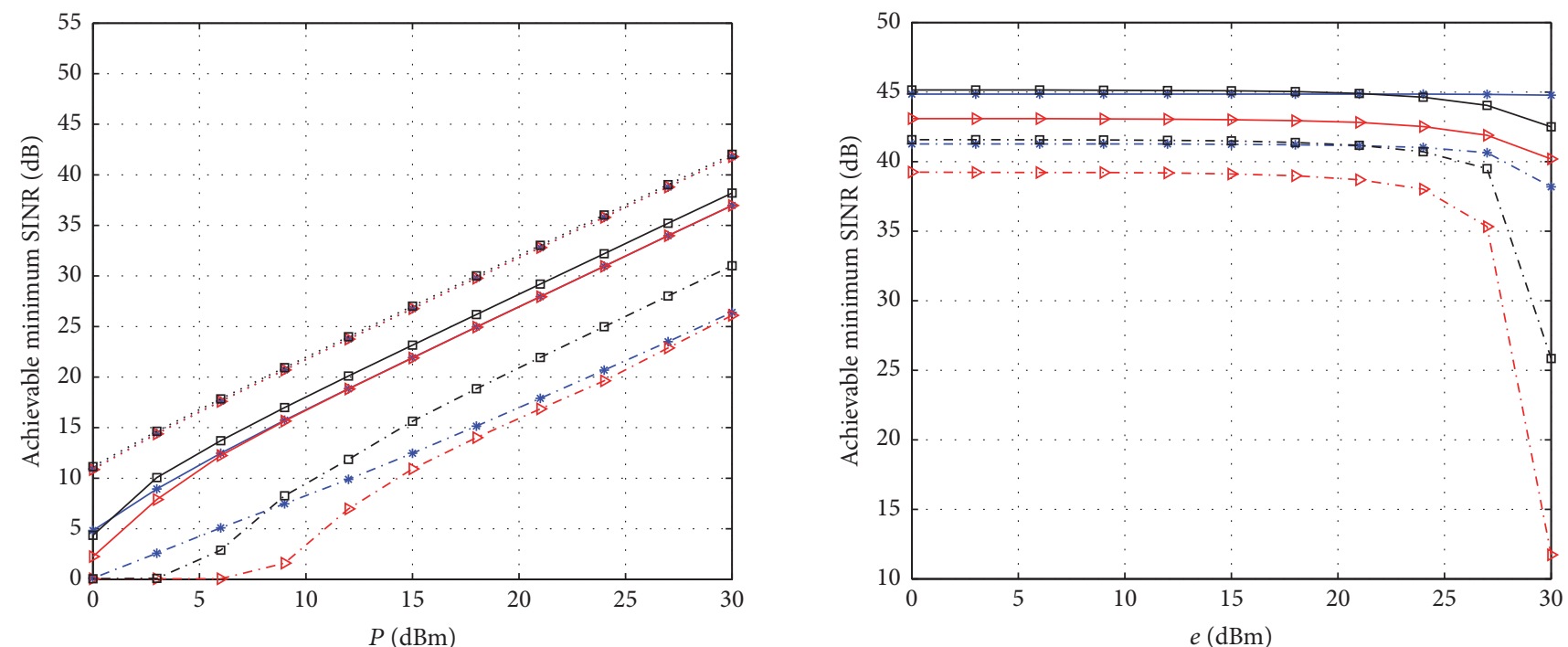

$$
\begin{aligned}
& \text { *.. SDR: } K=2, M=0, N=2 \\
& \text { D. ZF: } K=2, M=0, N=2 \\
& \text { ㅁ. ZF-DPC: } K=2, M=0, N=2 \\
& \rightarrow \text { SDR: } K=4, M=0, N=4 \\
& \rightarrow \text { ZF: } \quad K=4, M=0, N=4 \\
& \square \text { ZF-DPC: } K=4, M=0, N=4 \\
& \text { *- SDR: } K=8, M=0, N=8 \\
& \rightarrow-\mathrm{ZF}: \quad K=8, M=0, N=8 \\
& \text { - - ZF-DPC: } K=8, M=0, N=8
\end{aligned}
$$

FIGURE 7: Achievable minimum SINR versus transmit power with ID/EH users only.

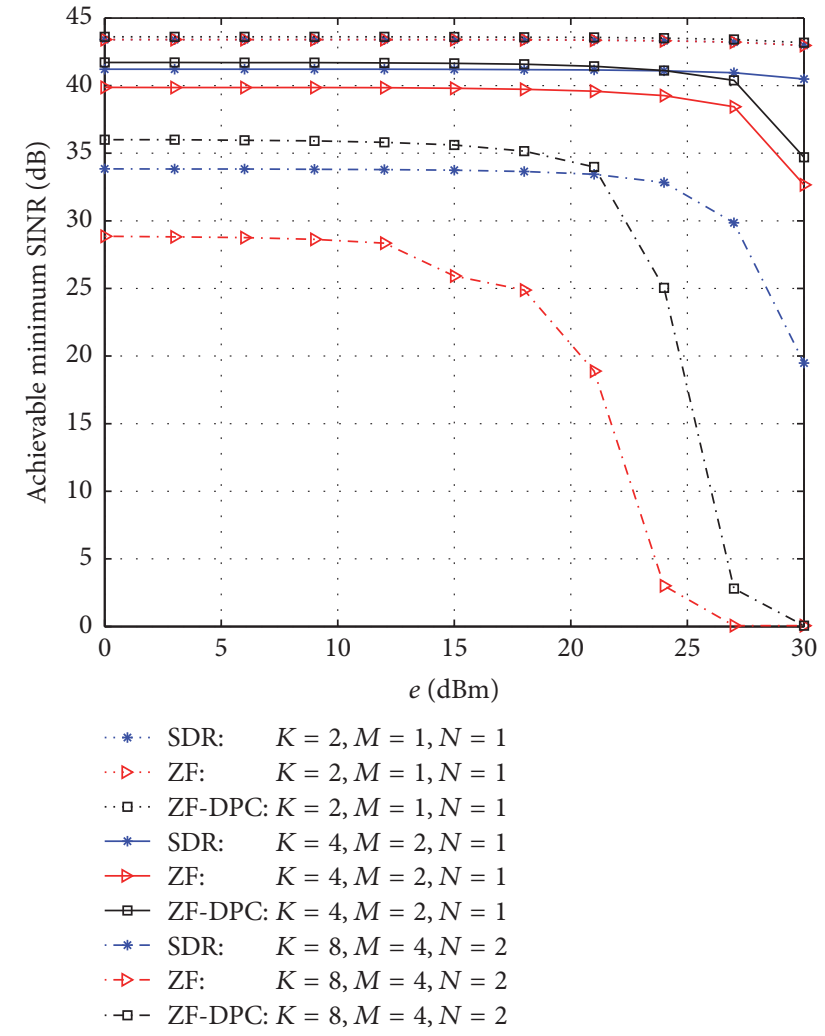

FIGURE 8: Achievable minimum SINR versus minimum EH constraint with different number of users.

$$
\begin{aligned}
& \text {-* SDR: } K=4, M=0, N=2 \\
& \rightarrow-\text { ZF: } \quad K=4, M=0, N=2 \\
& \text { - Z ZF-DPC: } K=4, M=0, N=2 \\
& \text { * SDR: } K=4, M=2, N=0 \\
& \rightarrow \text { ZF: } \quad K=4, M=2, N=0 \\
& \rightarrow \text { ZF-DPC: } K=4, M=2, N=0
\end{aligned}
$$

FIgURE 9: Achievable minimum SINR versus minimum EH constraint with fixed number of EH users.

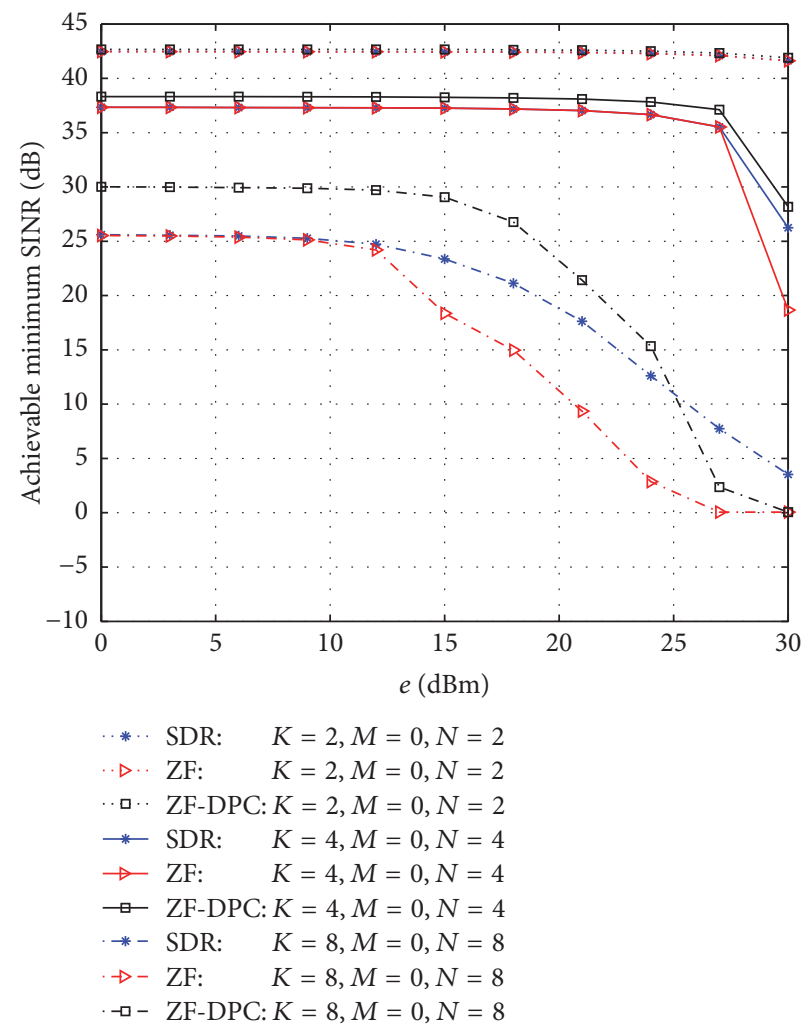

FIgURE 10: Achievable minimum SINR versus minimum EH constraint with ID/EH users only. 
that corresponds to the capacity of information transmission becomes lower.

\section{Conclusion}

In this paper, we, for the first time, studied the energy harvesting in the IoT for MISO SWIPT systems with heterogeneous users. We developed four schemes, namely, SDR, ZF, MRT, and ZF-DPC, to solve the nonconvex problem. We then compared them through simulations and provided suggestions on how to select the proper scheme in different scenarios. In the future work, we plan to solve the problems when there are multiple transmitters and the perfect CSI is not available.

\section{Appendix}

\section{A. The Proof of the Feasibility of Problem (11)}

For any given $\tau$, problem (11) can be reformulated as

$$
\begin{array}{ll}
\min _{\mathbf{v}, \boldsymbol{\rho}} & \sum_{k=1}^{K}\left\|\mathbf{v}_{k}\right\|^{2} \\
\text { s.t. } & \frac{\left|\mathbf{h}_{k}^{H} \mathbf{v}_{k}\right|^{2}}{\sum_{j \neq k}\left|\mathbf{h}_{k}^{H} \mathbf{v}_{j}\right|^{2}+\sigma_{k}^{2}} \geq \tau, \quad k=1, \ldots, M \\
& \frac{\rho_{k}\left|\mathbf{h}_{k}^{H} \mathbf{v}_{k}\right|^{2}}{\rho_{k} \sum_{j \neq k}\left|\mathbf{h}_{k}^{H} \mathbf{v}_{j}\right|^{2}+\rho_{k} \sigma_{k}^{2}+\sigma_{z}^{2}} \geq \tau, \\
& k=M+1, \ldots, M+N, \\
& \zeta_{k}\left(1-\rho_{k}\right)\left(\sum_{j=1}^{K}\left|\mathbf{h}_{k}^{H} \mathbf{v}_{j}\right|^{2}+\sigma_{k}^{2}\right) \geq e_{k}, \\
& k=M+1, \ldots, M+N, \\
& 0 \leq \rho_{k} \leq 1, \quad k=M+1, \ldots, M+N, \\
& \left(\sum_{j=1}^{K}\left|\mathbf{h}_{k}^{H} \mathbf{v}_{j}\right|^{2}+\sigma_{k}^{2}\right) \geq e_{k}, \\
& k=M+N+1, \ldots, K .
\end{array}
$$

Then examine whether the minimum power is less than the power constraint $P^{\max }$. Thus, we need to verify that problem (A.1) is feasible. And problem (A.1) is feasible if and only if the following problem is feasible:

$$
\begin{array}{ll}
\text { find } & \left\{\mathbf{v}_{k}, \rho_{k}\right\} \\
\text { s.t. } & \frac{\left|\mathbf{h}_{k}^{H} \mathbf{v}_{k}\right|^{2}}{\sum_{j \neq k}\left|\mathbf{h}_{k}^{H} \mathbf{v}_{j}\right|^{2}+\sigma_{k}^{2}} \geq \tau, \quad k=1, \ldots, M
\end{array}
$$

$$
\begin{gathered}
\frac{\rho_{k}\left|\mathbf{h}_{k}^{H} \mathbf{v}_{k}\right|^{2}}{\rho_{k} \sum_{j \neq k}\left|\mathbf{h}_{k}^{H} \mathbf{v}_{j}\right|^{2}+\rho_{k} \sigma_{k}^{2}+\sigma_{z}^{2}} \geq \tau, \\
k=M+1, \ldots, M+N, \\
0 \leq \rho_{k} \leq 1, \quad k=M+1, \ldots, M+N .
\end{gathered}
$$

First, it can be easily verified that if problem (A.2) is not feasible, then problem (A.1) cannot be feasible, since problem (A.1) has additional constraints on harvested power. Second, suppose that problem (A.2) is feasible, and let $\left\{\mathbf{v}_{k}\right\}$ and $\left\{\rho_{k}\right\}$ be a feasible solution. It can be shown that there always exists the new solution $\left\{\alpha \mathbf{v}_{k}\right\}, \alpha>1, \forall k$, with $\left\{\rho_{k}\right\}, k=$ $M+1, \ldots, M+N$, being feasible to problem (A.1). Thus to prove the feasibility of problem (A.1), we need to prove the feasibility of problem (A.2), which is feasible if and only if the following problem is feasible:

$$
\begin{aligned}
\text { find } & \left\{\mathbf{v}_{k}\right\} \\
\text { s.t. } & \frac{\left|\mathbf{h}_{k}^{H} \mathbf{v}_{k}\right|^{2}}{\sum_{j \neq k}\left|\mathbf{h}_{k}^{H} \mathbf{v}_{j}\right|^{2}+\sigma_{k}^{2}} \geq \tau, \quad k=1, \ldots, M, \\
& \frac{\left|\mathbf{h}_{k}^{H} \mathbf{v}_{k}\right|^{2}}{\sum_{j \neq k}\left|\mathbf{h}_{k}^{H} \mathbf{v}_{j}\right|^{2}+\sigma_{k}^{2}+\sigma_{z}^{2}} \geq \tau, \\
k & =M+1, \ldots, M+N .
\end{aligned}
$$

To prove the feasibility of problem (A.2), first, suppose that problem (A.3) is feasible, and let $\left\{\mathbf{v}_{k}\right\}$ denote a feasible solution to problem (A.3). Then, given any $0<\rho<1$, consider the following solution to problem (A.2): $\overline{\mathbf{v}}_{k}=$ $\mathbf{v}_{k} / \sqrt{\rho}, \forall k$, and $\bar{\rho}_{k}=\rho, k=M+1, \ldots, M+N$. We have

$$
\begin{gathered}
\frac{\left|\mathbf{h}_{k}^{H} \overline{\mathbf{v}}_{k}\right|^{2}}{\sum_{j \neq k}\left|\mathbf{h}_{k}^{H} \overline{\mathbf{v}}_{j}\right|^{2}+\sigma_{k}^{2}}=\frac{\left|\mathbf{h}_{k}^{H} \mathbf{v}_{k}\right|^{2}}{\sum_{j \neq k}\left|\mathbf{h}_{k}^{H} \mathbf{v}_{j}\right|^{2}+\rho \sigma_{k}^{2}} \\
>\frac{\left|\mathbf{h}_{k}^{H} \mathbf{v}_{k}\right|^{2}}{\sum_{j \neq k}\left|\mathbf{h}_{k}^{H} \mathbf{v}_{j}\right|^{2}+\sigma_{k}^{2}} \geq \tau, \quad k=1, \ldots, M,
\end{gathered}
$$

$$
\frac{\bar{\rho}_{k}\left|\mathbf{h}_{k}^{H} \overline{\mathbf{v}}_{k}\right|^{2}}{\bar{\rho}_{k} \sum_{j \neq k}\left|\mathbf{h}_{k}^{H} \overline{\mathbf{v}}_{j}\right|^{2}+\bar{\rho}_{k} \sigma_{k}^{2}+\sigma_{z}^{2}}
$$$$
=\frac{\left|\mathbf{h}_{k}^{H} \mathbf{v}_{k}\right|^{2}}{\sum_{j \neq k}\left|\mathbf{h}_{k}^{H} \mathbf{v}_{j}\right|^{2}+\rho \sigma_{k}^{2}+\sigma_{z}^{2}}
$$$$
>\frac{\left|\mathbf{h}_{k}^{H} \mathbf{v}_{k}\right|^{2}}{\sum_{j \neq k}\left|\mathbf{h}_{k}^{H} \mathbf{v}_{j}\right|^{2}+\sigma_{k}^{2}+\sigma_{z}^{2}} \geq \tau,
$$

$$
k=M+1, \ldots, M+N \text {. }
$$


Combining (A.4) with (A.5), we can see that $\left\{\overline{\mathbf{v}}_{k}\right\}$ and $\left\{\bar{\rho}_{k}\right\}$ are a feasible solution to problem (A.2). Therefore, if problem (A.3) is feasible, problem (A.2) must be feasible too. Second, suppose that problem (A.2) is feasible, and let $\left\{\mathbf{v}_{k}\right\}$ and $\left\{\rho_{k}\right\}$ denote a feasible solution to problem (A.2). Since $\rho_{k}<1, k=$ $M+1, \ldots, M+N$, we have

$$
\begin{aligned}
& \tau \leq \frac{\rho_{k}\left|\mathbf{h}_{k}^{H} \mathbf{v}_{k}\right|^{2}}{\rho_{k} \sum_{j \neq k}\left|\mathbf{h}_{k}^{H} \mathbf{v}_{j}\right|^{2}+\rho_{k} \sigma_{k}^{2}+\sigma_{z}^{2}} \\
&=\frac{\left|\mathbf{h}_{k}^{H} \mathbf{v}_{k}\right|^{2}}{\sum_{j \neq k}\left|\mathbf{h}_{k}^{H} \mathbf{v}_{j}\right|^{2}+\sigma_{k}^{2}+\sigma_{z}^{2} / \rho_{k}} \\
&<\frac{\left|\mathbf{h}_{k}^{H} \mathbf{v}_{k}\right|^{2}}{\sum_{j \neq k}\left|\mathbf{h}_{k}^{H} \mathbf{v}_{j}\right|^{2}+\sigma_{k}^{2}+\sigma_{z}^{2}}, \\
& \quad k=M+1, \ldots, M+N .
\end{aligned}
$$

As a result, $\left\{\mathbf{v}_{k}\right\}$ is also a feasible solution to problem (A.3). As problem (A.3) is a well-known SINR feasible problem, we can conclude that problem (11) is feasible. Then, given sufficiently small $\tau$, it always admits a feasible solution.

\section{B. The Proof of $\mathbf{X}_{k}^{*}$ Satisfying $\operatorname{rank}\left(\mathbf{X}_{k}^{*}\right)=1$}

The problem of maximizing the minimum SINR is equal to minimizing the total transmission power at BS which is also NP-hard. But, contrary to the QoS approach, it always admits a feasible solution, apart from the trivial case of zero channel vectors. By applying the bisection search method over a specific interval $[L, U]$, we have $\tau=(L+U) / 2$, and the problem is transformed into a QoS problem depicted in (B.1). If the minimum power is less than the power constraint $P^{\max }$, then update the lower bound $L=\tau$; otherwise update the upper bound $U=\tau$. Repeat the above steps until the algorithm stops when it satisfies $U-L \leq \varepsilon_{1}$, where $\varepsilon_{1}$ is the desired accuracy of the search.

$$
\begin{aligned}
\min _{\mathbf{X}, \boldsymbol{\rho}} & \sum_{k=1}^{K} \operatorname{Tr}\left(\mathbf{X}_{k}\right) \\
\text { s.t. } & \frac{\mathbf{h}_{k}^{H} \mathbf{X}_{k} \mathbf{h}_{k}}{\sum_{j \neq k} \mathbf{h}_{k}^{H} \mathbf{X}_{j} \mathbf{h}_{k}+\sigma_{k}^{2}} \geq \tau, \quad k=1, \ldots, M, \\
& \frac{\rho_{k} \mathbf{h}_{k}^{H} \mathbf{X}_{k} \mathbf{h}_{k}}{\rho_{k} \sum_{j \neq k} \mathbf{h}_{k}^{H} \mathbf{X}_{j} \mathbf{h}_{k}+\rho_{k} \sigma_{k}^{2}+\sigma_{z}^{2}} \geq \tau, \\
k & =M+1, \ldots, K,
\end{aligned}
$$

$$
\begin{aligned}
& \zeta_{k}\left(1-\rho_{k}\right)\left(\sum_{j=1}^{K} \mathbf{h}_{k}^{H} \mathbf{X}_{j} \mathbf{h}_{k}+\sigma_{k}^{2}\right) \geq e_{k}, \\
& \qquad k=M+1, \ldots, K, \\
& 0 \leq \rho_{k} \leq 1, \quad k=M+1, \ldots, K, \\
& \mathbf{X}_{k} \geq 0, \quad \forall k .
\end{aligned}
$$

After decoupling $\mathbf{X}_{k}$ and $\rho_{k}$, problem (B.1) can be reformulated as the following problem:

$$
\begin{array}{ll}
\min _{\mathbf{X}, \boldsymbol{\rho}} & \sum_{k=1}^{K} \operatorname{Tr}\left(\mathbf{X}_{k}\right) \\
\text { s.t. } \quad & \frac{1}{\tau} \mathbf{h}_{k}^{H} \mathbf{X}_{k} \mathbf{h}_{k}-\sum_{j \neq k} \mathbf{h}_{k}^{H} \mathbf{X}_{j} \mathbf{h}_{k} \geq \sigma_{k}^{2}, \\
& k=1, \ldots, M, \\
& \frac{1}{\tau} \mathbf{h}_{k}^{H} \mathbf{X}_{k} \mathbf{h}_{k}-\sum_{j \neq k} \mathbf{h}_{k}^{H} \mathbf{X}_{j} \mathbf{h}_{k} \geq \sigma_{k}^{2}+\frac{\sigma_{z}^{2}}{\rho_{k}}, \\
& k=M+1, \ldots, K, \\
& \sum_{j=1}^{K} \mathbf{h}_{k}^{H} \mathbf{X}_{j} \mathbf{h}_{k} \geq \frac{e_{k}}{\zeta_{k}\left(1-\rho_{k}\right)}-\sigma_{k}^{2}, \\
& 0 \leq \rho_{k} \leq 1, \quad k=M+1, \ldots, K, \\
& \mathbf{X}_{k} \geq 0, \quad \forall k .
\end{array}
$$

Let $\left\{\lambda_{k}\right\}$ and $\left\{\mu_{k}\right\}$ denote the dual variables associated with the SINR constraints and harvested power constraints of problem (B.2), respectively. Thus, we have $\lambda_{k} \geq 0, \forall k$, and $\mu_{k} \geq 0, k=$ $M+1, \ldots, K$. Then the Lagrangian [10] of problem (B.2) is defined as

$$
\begin{aligned}
& L\left(\left\{\mathbf{X}_{k}, \rho_{k}, \lambda_{k}, \mu_{k}\right\}\right) \triangleq \sum_{k=1}^{K}\left(\operatorname{Tr}\left(\mathbf{X}_{k}\right)-\operatorname{Tr}\left(\mathbf{X}_{k} \mathbf{S}_{k}\right)\right) \\
& -\sum_{k=1}^{M} \lambda_{k}\left(\frac{1}{\tau} \mathbf{h}_{k}^{H} \mathbf{X}_{k} \mathbf{h}_{k}-\sum_{j \neq k} \mathbf{h}_{k}^{H} \mathbf{X}_{j} \mathbf{h}_{k}-\sigma_{k}^{2}\right) \\
& -\sum_{k=M+1}^{K} \lambda_{k}\left(\frac{1}{\tau} \mathbf{h}_{k}^{H} \mathbf{X}_{k} \mathbf{h}_{k}-\sum_{j \neq k} \mathbf{h}_{k}^{H} \mathbf{X}_{j} \mathbf{h}_{k}-\sigma_{k}^{2}-\frac{\sigma_{z}^{2}}{\rho_{k}}\right) \\
& -\sum_{k=M+1}^{K} \mu_{k}\left(\sum_{j=1}^{K} \mathbf{h}_{k}^{H} \mathbf{X}_{j} \mathbf{h}_{k}-\frac{e_{k}}{\zeta_{k}\left(1-\rho_{k}\right)}+\sigma_{k}^{2}\right) .
\end{aligned}
$$


Then the dual function of problem (B.2) is given by

$$
\min _{0 \leq \rho_{k} \leq 1}\left\{\sum_{k=1}^{K}\left(\operatorname{Tr}\left(\mathbf{A}_{k} \mathbf{X}_{k}\right)-\operatorname{Tr}\left(\mathbf{S}_{k} \mathbf{X}_{k}\right)\right)+\sum_{k=1}^{K} \lambda_{k} \sigma_{k}^{2}+\sum_{k=M+1}^{K}\left(\frac{\lambda_{k} \sigma_{z}^{2}}{\rho_{k}}+\frac{\mu_{k} e_{k}}{\zeta_{k}\left(1-\rho_{k}\right)}\right)-\sum_{k=M+1}^{K} \mu_{k} \sigma_{k}^{2}\right\}
$$

where

$$
\begin{aligned}
\mathbf{A}_{k}= & \mathbf{I}_{N_{t}}+\sum_{j=1}^{K} \lambda_{j} \mathbf{h}_{j} \mathbf{h}_{j}^{H}-\sum_{j=M+1}^{K} \mu_{j} \mathbf{h}_{j} \mathbf{h}_{j}^{H} \\
& -\left(\frac{1}{\tau}+1\right) \lambda_{k} \mathbf{h}_{k} \mathbf{h}_{k}^{H}, \quad \forall k .
\end{aligned}
$$

Because the problem is feasible and the primal problem and the dual problem are strongly coupled, the duality gap is zero. Let $\left\{\lambda_{k}^{*}\right\},\left\{\mu_{k}^{*}\right\}$ denote the optimal dual solution to problem (B.2); accordingly, we define

$$
\begin{aligned}
\mathbf{A}_{k}^{*}= & \mathbf{I}_{N_{t}}+\sum_{j=1}^{K} \lambda_{j}^{*} \mathbf{h}_{j} \mathbf{h}_{j}^{H}-\sum_{j=M+1}^{K} \mu_{j}^{*} \mathbf{h}_{j} \mathbf{h}_{j}^{H} \\
& -\left(\frac{1}{\tau}+1\right) \lambda_{k}^{*} \mathbf{h}_{k} \mathbf{h}_{k}^{H}, \quad \forall k .
\end{aligned}
$$

According to the KKT conditions, we get

$$
\begin{aligned}
\mathbf{A}_{k}^{*}-\mathbf{S}_{k} & =0, \\
\mathbf{S}_{k} \mathbf{X}_{k}^{*} & =0,
\end{aligned}
$$

$\forall k$,

where the first equation is obtained by equating the gradient of the Lagrangian of problem (B.3) (with respect to $\mathbf{X}_{k}$ ) to zero and the second equation is the complementary condition for $\mathbf{X}_{k} \geq 0, \forall k$. From (B.7), we have

$$
\mathbf{A}_{k}^{*} \mathbf{X}_{k}^{*}=0, \quad \forall k .
$$

Moreover, it is observed from (B.4) that the optimal PS solution $\rho_{k}, k=M+1, \ldots, K$, must be the solution of the following problem:

$$
\begin{aligned}
\min _{\rho_{k}} & \frac{\lambda_{k}^{*} \sigma_{z}^{2}}{\rho_{k}}+\frac{\mu_{k}^{*} e_{k}}{\zeta_{k}\left(1-\rho_{k}\right)} \\
\text { s.t. } & 0 \leq \rho_{k} \leq 1, \quad k=M+1, \ldots, K .
\end{aligned}
$$

From (B.9), we can see that if $\lambda_{k}^{*}=0$ and $\mu_{k}^{*}>0$, the optimal solution will be $\rho_{k}^{*} \rightarrow 0$. Similarly, if $\mu_{k}^{*}=0$ and $\lambda_{k}^{*}>0$, then the optimal solution is $\rho_{k}^{*} \rightarrow 1$. Since $e_{k}>0, \tau>0, k=M+$ $1, \ldots, K$, the above two cases cannot happen. Next, we show that $\lambda_{k}^{*}=0$ and $\mu_{k}^{*}=0$ cannot be true for $k, k=M+1, \ldots, K$, by contradiction. Suppose that there exist some $k^{\prime} s$ such that $\lambda_{k}^{*}=\mu_{k}^{*}=0$. Define a set

$$
\Psi \triangleq\left\{k \mid \lambda_{k}^{*}=0, \mu_{k}^{*}=0, M+1 \leq k \leq K\right\},
$$

where $\Psi \neq \emptyset$. Define

$$
\mathbf{B}_{k}^{*}=\mathbf{I}_{N_{t}}+\sum_{j=1}^{K} \lambda_{j}^{*} \mathbf{h}_{j} \mathbf{h}_{j}^{H}-\sum_{j=M+1}^{K} \mu_{j}^{*} \mathbf{h}_{j} \mathbf{h}_{j}^{H} .
$$

Then $\mathbf{A}_{k}^{*}$ can be expressed as

$$
\mathbf{A}_{k}^{*}= \begin{cases}\mathbf{B}^{*}, & \text { if } k \in \Psi, \\ \mathbf{B}^{*}-\left(\frac{1}{\tau}+1\right) \lambda_{k}^{*} \mathbf{h}_{k} \mathbf{h}_{k}^{H}, & \text { otherwise. }\end{cases}
$$

Given $\mathbf{A}_{k}^{*} \succeq 0,-(1 / \tau+1) \lambda_{k}^{*} \mathbf{h}_{k} \mathbf{h}_{k}^{H} \preceq 0$, we have $\mathbf{B}^{*} \succeq 0$. In the following, we show that $\mathbf{B}^{*}>0$ by contradiction. Suppose that the minimum eigenvalue of $\mathbf{B}^{*} \geq 0$ is zero. Then, there exists at least $\mathbf{x} \neq 0$ such that $\mathbf{x}^{H} \mathbf{B}^{*} \mathbf{x}=0$. According to (B.12), it follows that

$$
\mathbf{x}^{H} \mathbf{A}_{k}^{*} \mathbf{x}=-\left(\frac{1}{\tau}+1\right) \lambda_{k}^{*} \mathbf{x}^{H} \mathbf{h}_{k} \mathbf{h}_{k}^{H} \mathbf{x} \geq 0, \quad k \notin \Phi .
$$

Note that when $k \notin \Psi$, we have $\lambda_{k}^{*}>0$; hence, according to (B.13), we get $\left|\mathbf{h}_{k}^{H} \mathbf{x}\right|^{2} \leq 0, k \notin \Psi$. It thus follows that

$$
\left|\mathbf{h}_{k}^{H} \mathbf{x}\right|^{2}=0, \quad k \notin \Psi .
$$

Thus, we have

$$
\begin{aligned}
\mathbf{x}^{H} \mathbf{B}^{*} \mathbf{x} & =\mathbf{x}^{H}\left(\mathbf{I}_{N_{t}}+\sum_{j=1}^{K} \lambda_{j}^{*} \mathbf{h}_{j} \mathbf{h}_{j}^{H}-\sum_{j=M+1}^{K} \mu_{j}^{*} \mathbf{h}_{j} \mathbf{h}_{j}^{H}\right) \mathbf{x} \\
& =\mathbf{x}^{H}\left(\mathbf{I}_{N_{t}}+\sum_{j=1}^{M} \lambda_{j}^{*} \mathbf{h}_{j} \mathbf{h}_{j}^{H}\right) \mathbf{x} \geq \mathbf{x}^{H} \mathbf{x}>0
\end{aligned}
$$

which contradicts $\mathbf{x}^{H} \mathbf{B}^{*} \mathbf{x}=0$. Thus we have $\mathbf{B}^{*}>0$; that is, $\operatorname{rank}\left(\mathbf{B}^{*}\right)=N_{t}$; then, from (B.12), we have $\operatorname{rank}\left(\mathbf{A}_{k}^{*}\right)=$ $N_{t}, k \in \Psi$. However, according to (B.8), we have $\mathbf{X}_{k}^{*}=0$; it is easily verified that $\mathbf{X}_{k}^{*}=0$ cannot be optimal for problem (B.1). Thus, we have

$$
\begin{aligned}
& \mathbf{B}_{k}^{*}=\mathbf{I}_{N_{t}}+\sum_{j=1}^{K} \lambda_{j}^{*} \mathbf{h}_{j} \mathbf{h}_{j}^{H}-\sum_{j=M+1}^{K} \mu_{j}^{*} \mathbf{h}_{j} \mathbf{h}_{j}^{H}, \\
& \mathbf{A}_{k}^{*}=\mathbf{B}^{*}-\left(\frac{1}{\tau}+1\right) \lambda_{k}^{*} \mathbf{h}_{k} \mathbf{h}_{k}^{H}, \quad k=M+1, \ldots, K .
\end{aligned}
$$

Because of $\operatorname{rank}\left(\mathbf{B}^{*}\right)=N_{t}$, it follows that $\operatorname{rank}\left(\mathbf{A}_{k}^{*}\right) \geq N_{t}-$ $1, k=M+1, \ldots, K$. We have seen that $\mathbf{X}_{k}^{*}=0$ is not optimal for problem (B.1); then we have $\operatorname{rank}\left(\mathbf{A}_{k}^{*}\right)=N_{t}-1, k=M+$ $1, \ldots, K$. As for the ID users $k, k=1, \ldots, M$, it is equivalent to $\mu_{k}^{*}=0, k=1, \ldots, M$. Then the proof is similar to the situation when $k=M+1, \ldots, K$. Thus, we can conclude that $\operatorname{rank}\left(\mathbf{X}_{k}^{*}\right)=1, \forall k$; then the proof is completed. 


\section{The Closed-Form Solution of ZF Scheme}

We can see from the bisection method that, for given $\tau$, the inner optimization problem is transformed into the problem of minimizing the total transmit power:

$$
\begin{array}{ll}
\min _{p_{k}, \rho} & \sum_{k=1}^{K} p_{k} \\
\text { s.t. } \quad & G_{k, k} p_{k} \geq \tau \sigma_{k}^{2}, \quad k=1, \ldots, M, \\
& G_{k, k} p_{k} \geq \tau \sigma_{k}^{2}+\frac{\tau \sigma_{z}^{2}}{\rho_{k}}, \\
& \quad k=M+1, \ldots, M+N, \\
& \zeta_{k}\left(1-\rho_{k}\right)\left(G_{k, k} p_{k}+\sigma_{k}^{2}\right) \geq e_{k}, \\
& \quad k=M+1, \ldots, M+N, \\
& \zeta_{k}\left(G_{k, k} p_{k}+\sigma_{k}^{2}\right) \geq e_{k}, \\
& k=M+N+1, \ldots, K,
\end{array}
$$

$p_{k} \geq 0, \quad \forall k$

Now the variables $p_{k}$ and $\rho_{k}$ of user $k$ are decoupled and do not appear in other users' constraints. Hence, problem (C.1) can be decomposed into $K$ subproblems as follows:

$$
\begin{array}{ll}
\min _{p_{k}} & p_{k} \\
\text { s.t. } & G_{k, k} p_{k} \geq \tau \sigma_{k}^{2}, \quad k=1, \ldots, M, \\
& p_{k} \geq 0, \quad k=1, \ldots, M, \\
\min _{p_{k}, \rho_{k}} & p_{k} \\
& \\
\text { s.t. } & \frac{1}{\tau} G_{k, k} p_{k} \geq \sigma_{k}^{2}+\frac{\sigma_{z}^{2}}{\rho_{k}}, \\
& \\
& \\
& \zeta_{k}\left(1-\rho_{k}\right)\left(G_{k, k} p_{k}+\sigma_{k}^{2}\right) \geq e_{k}, \\
& \quad k=M+1, \ldots, M+N, \ldots, M+N, \\
& 0 \leq \rho_{k} \leq 1, \quad k=M+1, \ldots, M+N, \\
& p_{k} \geq 0, \quad k=M+1, \ldots, M+N, \\
& p_{k} \quad k=M+N+1, \ldots, K, \\
& p_{p_{k}} \geq 0, \quad k=M+N+1, \ldots, K . \\
\text { s.t. } & \zeta_{k}\left(G_{k, k} p_{k}+\sigma_{k}^{2}\right) \geq e_{k}, \\
&
\end{array}
$$

For problem (C.2), we have $p_{k}^{*}=\tau \sigma_{k}^{2} / G_{k, k}, k=1, \ldots, M$. And, for problem (C.4), we have $p_{k}^{*}=\left(\gamma-\sigma_{k}^{2}\right) / G_{k, k}, k=$ $M+N+1, \ldots, K$. Then, we show that, for problem (C.3), with the optimal solution $p_{k}^{*}$ and $\rho_{k}^{*}$, the SINR constraint and harvested power constraint should both hold with equality by contradiction. First, suppose that both the two SINR and harvested power constraints are not tight, given the solution $\bar{p}_{k}^{*}, \bar{\rho}_{k}^{*}$. Thus, there must exist an $\alpha_{k}, 0<\alpha_{k}<1$, such that, with the new solution $p_{k}=\alpha_{k} \bar{p}_{k}^{*}, \rho_{k}=\bar{\rho}_{k}^{*}$, either the SINR or harvested power constraint is tight. Moreover, with this new solution, the transmission power is reduced, which contradicts the fact that $\bar{p}_{k}^{*}$ and $\bar{\rho}_{k}^{*}$ are optimal for problem (C.3). Thus, the case where both the SINR and harvested power constraints are not tight is not valid. Next, consider the case when the SINR constraint is tight but the harvested power constraint is not tight. In this case, we can increase the value of $\bar{\rho}_{k}^{*}$ by a sufficiently small amount such that both the SINR and harvested power constraints become nontight. Then, we can conclude that this case cannot be true either. Similarly, we can show that the case where the harvested power constraint is tight but the SINR constraint is not tight cannot be true. To summarize, the SINR and harvested power constraints must both hold with equality. Hence, we have the following equation:

$$
\rho_{k}=\frac{\tau \sigma_{z}^{2}}{G_{k, k} p_{k}-\tau \sigma_{k}^{2}}=1-\frac{e_{k}}{\zeta_{k}\left(G_{k, k} p_{k}+\sigma_{k}^{2}\right)}
$$

Substituting $x_{i}, \alpha, \beta$, and $\gamma$ into (C.5), we can get

$$
\rho_{i}=1-\frac{\gamma}{x_{i}}=\frac{\beta}{x_{i}-\alpha} .
$$

Then, there are two distinct solutions:

$$
\begin{aligned}
& x_{1}=\frac{1}{2}\left(\alpha+\beta+\gamma-\sqrt{(\alpha+\beta+\gamma)^{2}-4 \alpha \gamma}\right), \\
& x_{2}=\frac{1}{2}\left(\alpha+\beta+\gamma+\sqrt{(\alpha+\beta+\gamma)^{2}-4 \alpha \gamma}\right) .
\end{aligned}
$$

Given the existence of ID/EH users, $0<\rho_{k}<1$ should be satisfied. Thus, according to (C.6), we have $x_{i}>\gamma$ and $x_{i}>\alpha+\beta$; that is, $x_{i}>\max (\gamma, \alpha+\beta)$. We can easily show that $x_{1}<\max (\gamma, \alpha+\beta)$ and $x_{2}>\max (\gamma, \alpha+\beta)$, which imply that the optimal solution is $x^{*}=x_{2}$. Thus, we can derive the optimal solution $p_{k}^{*}=\left(1 / G_{k, k}\right)\left(x^{*}-\sigma_{k}^{2}\right)$ and $\rho_{k}^{*}=1-\gamma / x^{*}$. Then, check whether the constraint $\sum_{k=1}^{K} p_{k}^{*} \leq P^{\max }$ is tight; if it is not, update until the constraint is tight. 


\section{Notations}

Boldface uppercase letter X: Matrix

Boldface lowercase letter $\mathbf{x}$ : Column vector

Lowercase letter $x$ :

Scalar

S:

$\mathbf{S}^{1 / 2}$ :

$\operatorname{trace}(\mathbf{S})$ :

$\mathbf{S} \geq 0$ :

A:

$\operatorname{rank}(\mathbf{A})$ :

$\mathbf{A}^{*}$ :

$\mathbf{A}^{T}$ :

$\mathbf{A}^{H}$ :

$\operatorname{diag}\left\{x_{1}, x_{2}, \ldots, x_{M}\right\}$

$\mathbf{I}_{n}:$

$\|\cdot\|$ :

$|\cdot|:$

$\mathbf{x} \sim \mathscr{C} \mathscr{N}\left(\mu, \sigma^{2}\right)$

“ ":

$\mathbb{C}^{m \times n}:$

Square matrix

Square root of $\mathbf{S}$

The trace of $\mathbf{S}$

matrix

The rank of $\mathbf{A}$

Transpose of $\mathbf{A}$

transpose of $\mathbf{A}$

complex vector

complex scalar
$\mathbf{S}$ is a positive semidefinite

Arbitrary-size matrix A

Complex conjugate of $\mathbf{A}$

Hermitian (conjugate)

$M \times M$ diagonal matrix with

$x_{1}, x_{2}, \ldots, x_{M}$ being the

diagonal elements

$n \times n$ identity matrix

The Euclidean norm of a

The absolute value of a

The distribution of a circularly symmetric complex

Gaussian (CSCG) random vector $\mathbf{x}$ with mean $\mu$ and covariance matrix $\sigma^{2}$ Stands for "distributed as" The space of $m \times n$ complex matrices.

\section{Conflicts of Interest}

The authors declare that they have no conflicts of interest.

\section{Acknowledgments}

This work was supported in part by the National Natural Science Foundation of China under Grant no. 61471408, in part by the Fundamental Research Funds for the Central Universities under Grant no. 2016YXMS293, and in part by CCF-Tencent Grant no. IAGR20160106.

\section{References}

[1] D. Minoli, K. Sohraby, and B. Occhiogrosso, "IoT considerations, requirements, and architectures for smart buildings energy optimization and next generation building management systems," IEEE Internet of Things Journal, vol. 4, no. 1, pp. 269283, Feb 2017.

[2] J. Gubbi, R. Buyya, S. Marusic, and M. Palaniswami, "Internet of Things (IoT): a vision, architectural elements, and future directions," Future Generation Computer Systems, vol. 29, no. 7, pp. 1645-1660, 2013.

[3] S. Oh, J. Lee, and S. Park, "Energy efficient and accurate monitoring of large-scale diffusive objects in internet of things," IEEE Communications Letters, vol. 21, no. 3, pp. 612-615, 2017.
[4] H. Ju and R. Zhang, "Throughput maximization in wireless powered communication networks," IEEE Transactions on Wireless Communications, vol. 13, no. 1, pp. 418-428, 2014.

[5] L. Liu, R. Zhang, and K. C. Chua, "Multi-antenna wireless powered communication with energy beamforming," IEEE Transactions on Communications, vol. 62, no. 12, pp. 4349-4361, 2014.

[6] Y. Che, J. Xu, L. Duan, and R. Zhang, "Multiantenna wireless powered communication with cochannel energy and information transfer," IEEE Communications Letters, vol. 19, no. 12, pp. 2266-2269, 2015.

[7] B. Xu, Y. Zhu, and R. Zhang, "Optimal power allocation for a two-link interference channel with SWIPT," in Proceedings of the 6th International Conference on Wireless Communications and Signal Processing (WCSP '14), pp. 1-5, IEEE, Hefei, China, October 2014.

[8] R. Zhang and C. K. Ho, "MIMO broadcasting for simultaneous wireless information and power transfer," IEEE Transactions on Wireless Communications, vol. 12, no. 5, pp. 1989-2001, 2013.

[9] X. Zhou, R. Zhang, and C. K. Ho, "Wireless information and power transfer: architecture design and rate-energy tradeoff," IEEE Transactions on Communications, vol. 61, no. 11, pp. 47544761, 2013.

[10] S. Timotheou, I. Krikidis, G. Zheng, and B. Ottersten, "Beamforming for MISO interference channels with QoS and RF energy transfer," IEEE Transactions on Wireless Communications, vol. 13, no. 5, pp. 2646-2658, 2014.

[11] S. Timotheou, I. Krikidis, and B. Ottersten, "MISO interference channel with QoS and RF energy harvesting constraints," in Proceedings of the IEEE International Conference on Communications (ICC '13), pp. 4191-4196, IEEE, Budapest, Hungary, June 2013.

[12] Q. Shi, L. Liu, W. Xu, and R. Zhang, "Joint transmit beamforming and receive power splitting for MISO SWIPT systems," IEEE Transactions on Wireless Communications, vol. 13, no. 6 , pp. 3269-3280, 2014.

[13] M.-M. Zhao, Y. Cai, Q. Shi, B. Champagne, and M.-J. Zhao, "Robust transceiver design for miso interference channel with energy harvesting," IEEE Transactions on Signal Processing, vol. 64, no. 17, pp. 4618-4633, 2016.

[14] Z. Q. Luo, W. Ma, S. A. M. C et al., "Semidefinite relaxation of quadratic optimization problems," IEEE Signal Processing Magazine, vol. 64, no. 17, pp. 4618-4633, Sept 2010.

[15] H. Weingarten, Y. Steinberg, and S. Shamai, "The capacity region of the Gaussian multiple-input multiple-output broadcast channel," Institute of Electrical and Electronics Engineers. Transactions on Information Theory, vol. 52, no. 9, pp. 39363964, 2006.

[16] G. Caire and S. Shamai, "On the achievable throughput of a multiantenna Gaussian broadcast channel," Institute of Electrical and Electronics Engineers. Transactions on Information Theory, vol. 49, no. 7, pp. 1691-1706, 2003.

[17] K. Xu, Y. Qu, and K. Yang, "A tutorial on the internet of things: From a heterogeneous network integration perspective," IEEE Network, vol. 30, no. 2, pp. 102-108, 2016.

[18] F. Saffre, "Tutorial ii: the green internet of things," in Proceedings of the 11th International Conference on Innovations in Information Technology (IIT '15), pp. XXXVII-XXXVII, Dubai, United Arab Emirates, November 2015.

[19] C. Park, "A Secure and efficient ECQV implicit certificate issuance protocol for the internet of things applications," IEEE Sensors Journal, vol. 17, no. 7, pp. 2215-2223, 2017. 
[20] J. Xu, L. Liu, and R. Zhang, "Multiuser miso beamforming for simultaneous wireless information and power transfer," IEEE Transactions on Signal Processing, vol. 62, no. 18, pp. 4778-4810, 2014.

[21] L. Liu, R. Zhang, and K.-C. Chua, "Wireless information transfer with opportunistic energy harvesting," IEEE Transactions on Wireless Communications, vol. 12, no. 1, pp. 288-300, 2013.

[22] B. Xu, Y. Zhu, and R. Zhang, "Optimized power allocation for interference channel with SWIPT," IEEE Wireless Communications Letters, vol. 5, no. 2, pp. 220-223, 2016.

[23] N. D. Sidiropoulos, T. N. Davidson, and Z.-Q. Luo, “Transmit beamforming for physical-layer multicasting," IEEE Transactions on Signal Processing, vol. 54, no. 6 I, pp. 2239-2251, 2006.

[24] S. Boyd and L. Vandenberghe, Convex Optimization, Cambridge University Press, 2004.

[25] M. Grant and S. Boyd, "Cvx: Matlab software for disciplined convex programming, version 2.0 beta," Sept 2012.

[26] B. Gopalakrishnan and N. D. Sidiropoulos, "High performance adaptive algorithms for single-group multicast beamforming," IEEE Transactions on Signal Processing, vol. 63, no. 16, pp. 43734384, 2015.

[27] J. Lei, Z. Han, M. A. Vazquez-Castro, and A. Hjorungnes, "Secure satellite communication systems design with individual secrecy rate constraints," IEEE Transactions on Information Forensics and Security, vol. 6, no. 3, pp. 661-671, 2011.

[28] Z. Shen, R. Chen, J. G. Andrews, R. W. Heath Jr., and B. L. Evans, "Low complexity user selection algorithms for multiuser MIMO systems with block diagonalization," IEEE Transactions on Signal Processing, vol. 54, no. 9, pp. 3658-3663, 2006.

[29] E. A. Jorswieck, E. G. Larsson, and D. Danev, "Complete characterization of the Pareto boundary for the miso interference channel," IEEE Transactions on Signal Processing, vol. 56, no. 10, part 2, pp. 5292-5296, 2008.

[30] F. Alizadeh and D. Goldfarb, "Second-order cone programming," Mathematical Programming-Series B, vol. 95, pp. 3-51, 2003.

[31] Z. Luo, M. Zhao, S. Liu, and Y. Liu, "Greville-to-Inverse-Freville algorithm for V-BLAST systems," in Proceedings of the IEEE International Conference on Communications (ICC '06), pp. 4214-4218, Istanbul, Turkey, July 2006.

[32] R. A. Horn and C. R. Johnson, Matrix Analysis, Cambridge university press, 2012.

[33] F. Rashid-Farrokhi, "Joint optimal power control and beamforming in wireless networks using antenna arrays," IEEE Transactions on Communications, vol. 46, no. 10, pp. 1313-1324, 1998. 


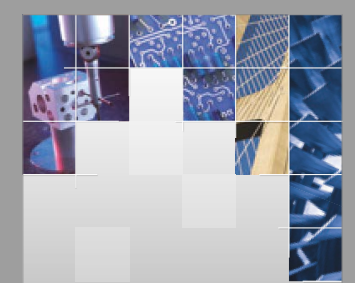

\section{Enfincering}
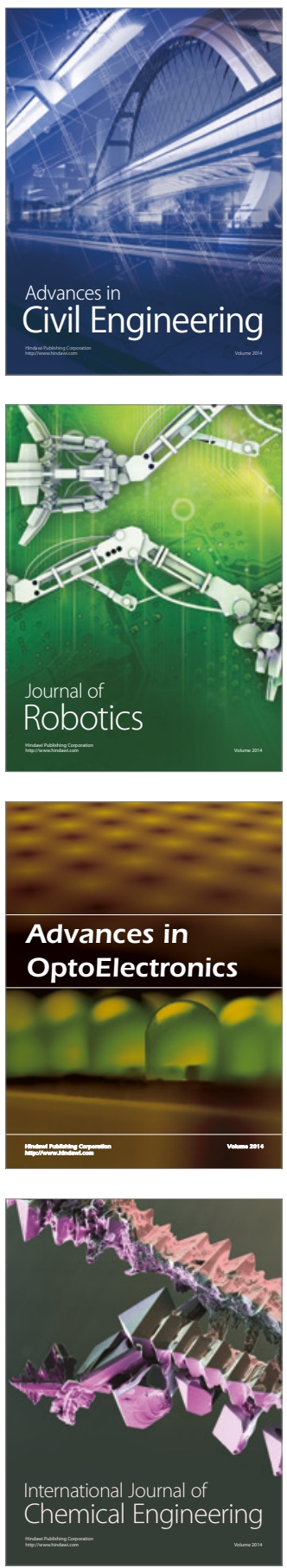

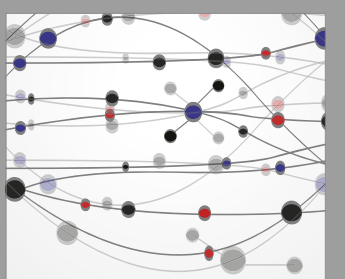

The Scientific World Journal

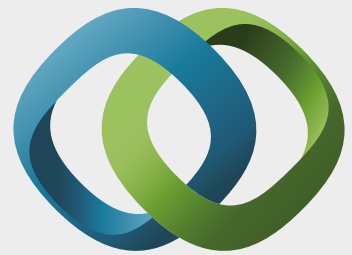

\section{Hindawi}

Submit your manuscripts at

https://www.hindawi.com
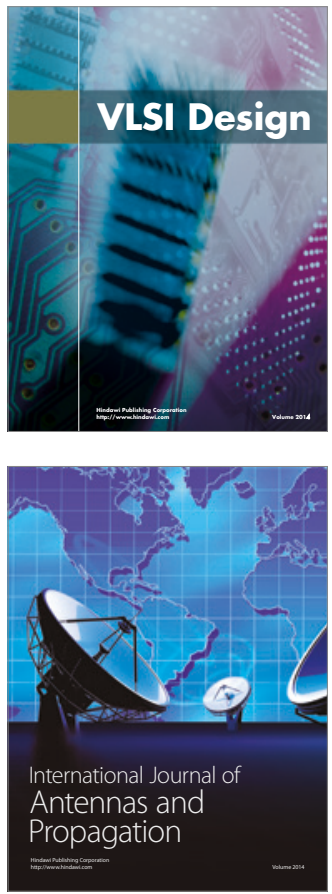

\section{Rotating}

Machinery
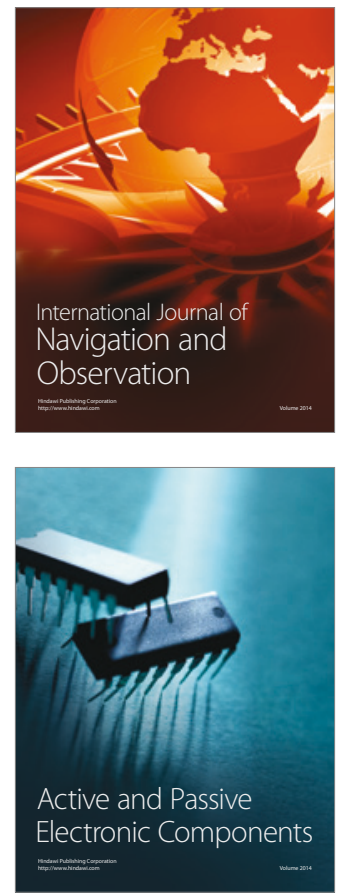
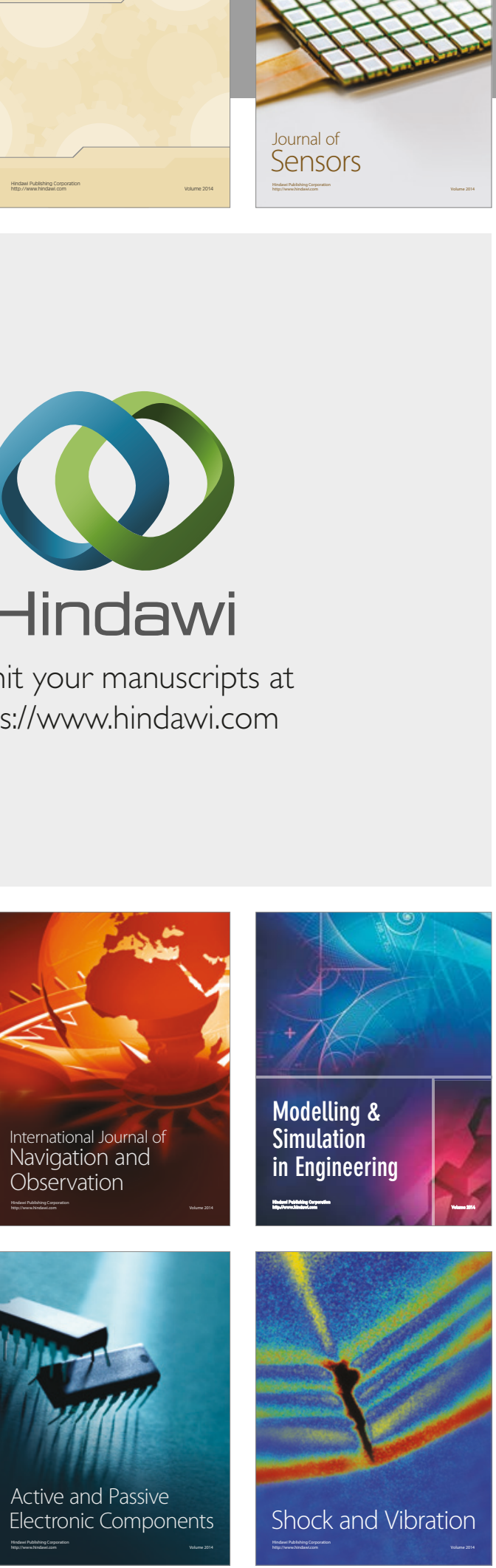
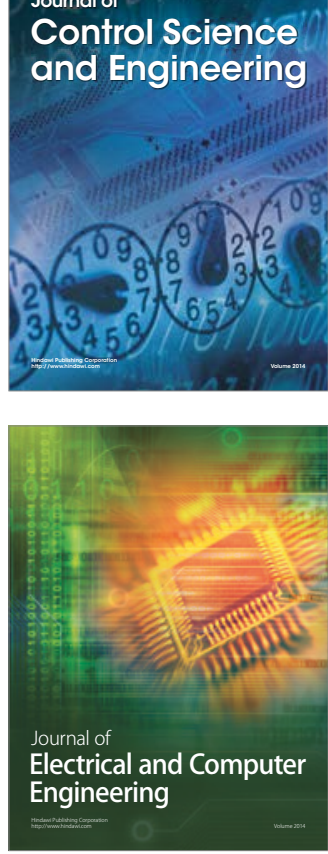

Distributed

Journal of

Control Science

and Engineering
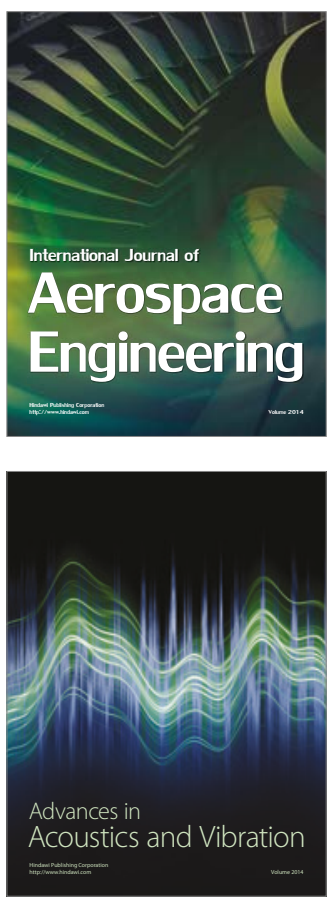

Sensor Networks 